\title{
Variations on U-shaped learning
}

\author{
Lorenzo Carlucci, ${ }^{\mathrm{a}, 1}$ Sanjay Jain, ${ }^{\mathrm{b}, 2}$ Efim Kinber $^{\mathrm{c}}$ and \\ Frank Stephan d,3
}

\author{
${ }^{a}$ Department of Computer and Information Sciences, University of Delaware, \\ Newark, DE 19716-2586, U.S.A. and Dipartimento di Matematica, Università di \\ Siena, Pian dei Mantellini 44, 00153, Siena, Italy \\ ${ }^{\mathrm{b}}$ School of Computing, National University of Singapore, Singapore 117543, \\ Republic of Singapore \\ c Department of Computer Science, Sacred Heart University, Fairfield, CT \\ 06432-1000, U.S.A. \\ ${ }^{\mathrm{d}}$ School of Computing and Department of Mathematics, National University of \\ Singapore, 3 Science Drive 2, Singapore 117543, Republic of Singapore
}

\begin{abstract}
The paper deals with the following problem: is returning to wrong conjectures necessary to achieve full power of algorithmic learning? Returning to wrong conjectures complements the paradigm of $U$-shaped learning $[3,7,9,24,29]$ when a learner returns to old correct conjectures. We explore our problem for classical models of learning in the limit from positive data: explanatory learning (when a learner stabilizes in the limit on a correct grammar) and behaviourally correct learning (when a learner stabilizes in the limit on a sequence of correct grammars representing the target concept). In both cases we show that returning to wrong conjectures is necessary to achieve full learning power. In contrast, one can modify learners (without losing learning power) such that they never show inverted U-shaped learning behaviour, that is, never return to old wrong conjecture with a correct conjecture in-between. Furthermore, one can also modify a learner (without losing learning power) such that it does not return to old "overinclusive" conjectures containing non-elements of the target language. We also consider our problem in the context of vacillatory learning (when a learner stabilizes on a finite number of correct grammars) and show that each of the following four constraints is restrictive (that is, reduces learning power): the learner does not return to old wrong conjectures; the learner is not inverted U-shaped; the learner does not return to old overinclusive conjectures; the learner does not return to old overgeneralizing conjectures. We also show that learners that are consistent with the input seen so far can be made decisive [3,25]: on any text, they do not return to any old conjectures - wrong or right.
\end{abstract}




\section{Introduction}

$U$-shaped learning is a well-known pattern of learning behaviour in which the learner first learns the correct target behaviour, later abandons it, and finally returns to the correct target behaviour once again. The phenomenon of U-shaped learning has been observed by cognitive and developmental psychologists in many different cases of child development, such as language learning $[7,24,29]$, understanding of temperature [29,30] and face recognition [8]. The ability of models of human learning to accommodate U-shaped learning has progressively become one of the important criteria of their adequacy; see $[24,26]$ and the recent [31]. Renewed interest in U-shaped learning is also witnessed by the fact that the Journal of Cognition and Development dedicated its first issue in the year 2004 to this phenomenon.

Cognitive and developmental psychology deals primarily with the problem of designing models of learning that adequately accommodate U-shaped behaviour. Baliga, Case, Merkle, Stephan, and Wiehagen [3] who initiated the study of U-shaped learning in the context of Gold-style algorithmic learning, asked a different question: is U-shaped behaviour really necessary for full learning power? In particular, they showed that U-shaped behaviour is avoidable for so-called TxtEx-learning (explanatory learning) — when the learner stabilizes in the limit on a single correct conjecture. This result contrasts with the result by Fulk, Jain, and Osherson [17] who demonstrated that U-shaped learning is necessary for the full power of so-called TxtBc-learners (behaviourally correct learners) that stabilize on a (possibly infinite) sequence of different grammars representing the target language. In a sequel paper [9], Carlucci, Case, Jain, and Stephan investigated U-shaped behaviour with respect to the model of vacillatory (or TxtFex) learning, where the learner is required to stabilize on a finite number of correct conjectures. Vacillatory learning, introduced by Case [11], features a hierarchy of more and more powerful learning criteria between TxtEx and TxtBc identification. It was shown in [9] that disallowing U-shaped behaviour for vacillatory learners makes the whole hierarchy collapse to simple TxtEx-learning, i.e. nullifies the extra power of allowing vacillation between a finite number of conjectures.

The U-shaped pattern of learning that we have discussed so far (i.e., a correctincorrect-correct pattern) is only a species of a more general learning behaviour, that also goes under the name of U-shaped learning. This more general meaning of U-shaped learning is explicitly introduced, for example, at

Email addresses: carlucci5@unisi.it (Lorenzo Carlucci,), sanjay@comp.nus.edu.sg (Sanjay Jain,), kinbere@sacredheart.edu (Efim Kinber), fstephan@comp.nus.edu.sg (Frank Stephan).

1 Supported in part by NSF grant number NSF CCR-0208616.

2 Supported in part by NUS grant number R252-000-127-112.

3 Supported in part by NUS grant number R252-000-212-112. 
the beginning of [29], the main reference in the psychological literature for the study of U-shaped behaviour. In this more general sense, U-shaped learning refers to any learning behaviour in which the learner first adopts some (not necessarily correct) behaviour, then abandons it, and, later, returns to it once again. The original interest in this phenomena, for developmental psychologists, is their "non-monotonic" character. Some particular cases of this general kind of U-shaped learning have been recently experimentally documented by developmental psychologists in the context of infants' face recognition. For example, it has been shown that children exhibit an "inverted-U-shaped" learning curve (a wrong-correct-wrong pattern) for recognition of inverted faces and an "N-shaped" learning curve (a wrong-correct-wrong-correct pattern) for recognition of upright faces $[14,15]$.

Both these examples of U-shaped behaviour feature return to a wrong previously abandoned behaviour. This kind of learning behaviour is, prima facie, definitely less reasonable than returning to previous correct conjectures. If a learner returns to a correct conjecture that the learner has previously abandoned, it is, of course, dictated by the goal of correctly learning the target concept. On the other hand, when a learner returns to a previously abandoned wrong conjecture, this is not desirable if a learner wants to be efficient.

Partially motivated by the experimental findings mentioned above, in the present paper we study the following general question: if and when returning to wrong conjectures is necessary for the full power of computational learnability?

In particular, we answer the latter question in the context of the following main models.

(a) a model in which a learner cannot return to any previously abandoned wrong conjecture;

(b) a model in which a learner cannot return to a previously abandoned conjecture that is "overinclusive" in the sense of containing elements not belonging to the target concept.

We also study the following less restrictive natural variant of model (a).

$\left(a^{\prime}\right)$ a model in which a learner cannot return to a previously abandoned wrong conjecture if a correct conjecture has been made in-between.

Model $\left(\mathrm{a}^{\prime}\right)$ is directly inspired by the above-mentioned concrete cases of inverted-U-shaped and N-shaped behaviour documented in the psychological literature $[14,15]$. It also represents the exact inverse of the original non Ushaped model studied in $[3,9]$.

Finally, we study the following natural variant of (b).

$\left(b^{\prime}\right)$ a model in which a learner cannot return to a previously abandoned conjecture that "overgeneralizes" in the sense of being a proper superset of the target language. 
The latter model is motivated by the fact that overgeneralization is one of the major concerns in the study of learning behaviour [24] (note also that Karl Popper, see [27], considered refutation of overgeneralizing conjectures as an important part of learning and scientific discovery processes). It is also interesting to observe how overgeneralization plays a role at the technical level as well: one can note in fact that the necessary overgeneralization of learning machines is an essential ingredient in the proof showing that U-shaped behaviour is unavoidable for the full learning power of TxtBc-learning (see $[17,3])$, as well as in our proof of Theorem 36 .

We compare the new models with regular types of learning in the limit and provide a full answer to the following question: when and how is returning to wrong conjectures necessary? The results that we obtained lead us to the following general conclusions. If we take TxtEx or TxtBc identification as a model of learning behaviour, then, returning to previously abandoned wrong conjectures is necessary to achieve full power of learnability; however, inverted U-shapes are redundant and it is not necessary to return to old overinclusive conjectures or to old overgeneralizing conjectures. On the other hand, for vacillatory identification, returning to wrong conjectures, inverted U-shapes and returning to overinclusive conjectures are all necessary in a very strong sense: disallowing this kind of U-shapes collapses the whole TxtFex-hierarchy to simple TxtEx-learning. In contrast, if returning to previously conjectured proper supersets is disallowed, no such collapse occurs, but, still, returning to such overgeneralizing conjectures is necessary for full learning power at each level of the vacillatory hierarchy. We compare more thoroughly these conclusions with results from [9] on returning to correct conjectures.

While being admittedly less central than TxtEx and TxtBc, we believe that vacillatory learning (introduced in [11]) is both an interesting candidate for a model of human learning, and a mathematically natural formal criterion. It has often been observed that syntactic convergence as featured in TxtEx might be a too restrictive condition for human learning, while allowing convergence to infinitely many correct conjectures as in TxtBc-learning might be a too liberal condition. The hierarchy of vacillatory learning criteria sits strictly between those two extremes. Also, in the study of U-shaped learning, the vacillatory hierarchy has shown to exhibit non-trivial properties that distinguish it from both Ex and TxtBc. For example, the study of non U-shaped learning in [9] gave rise to an interesting and quite rare phenomenon in algorithmic learning theory: that one parameter-free criterion covers the second but not the third level of some hierarchy. This example is that all classes in $\mathbf{T x t F e x}_{2}$ can be TxtBc-learned without U-shapes, but this is no longer true for some classes in $\mathbf{T x t F e x}_{3}$. We will show that this difference does not hold when returning to wrong conjectures is considered (see Theorem 34).

The present paper has the following structure. Section 2 contains necessary notations and basic definitions. Section 3 contains definitions of all variants of previously known models of non U-shaped learning, as well as the models 
introduced in the present paper. In Section 4 we explore our variants of non Ushaped learning in the context of TxtEx-learning — when learners stabilize on one correct grammar for the target language. First we show that returning to wrong conjectures is necessary for the full power of TxtEx-learning. To prove this result, we establish that learners not returning to wrong conjectures are as powerful as so-called decisive learners - the ones that never return to any previously abandoned conjecture, wrong or right (Theorem 21). Decisive learners are known [3] to be generally weaker than general TxtEx-learners. On the other hand, any TxtEx-learner can be replaced by a learner not returning to overinclusive conjectures (Theorem 22). From this result we also obtain that any TxtEx-learner can be replaced by a learner not returning to overgeneralizing conjectures and by a learner not showing an inverted-U-shaped behaviour as well (Corollaries 23 and 25 respectively).

In Section 5 we consider our four variants of non U-shaped learning in the context of the vacillatory learning criteria $\mathbf{T x t F e x}_{b}$ — when a learner stabilizes on no more than $b$ grammars describing the target language. The vacillatory criteria form a hierarchy of more and more powerful learning criteria strictly between TxtEx and TxtBc, increasing in the parameter $b$. The more vacillation is allowed, the more learning power is possible [11]. We extend a result of Section 4 to show that vacillatory learners without returning to wrong conjectures do no better than just decisive TxtEx-learners. As for vacillatory learners not returning to overinclusive conjectures and for vacillatory learners that do not show an inverted-U-shaped behaviour, they turn out to be doing no better than regular TxtEx-learners with the same restrictions. It was shown in [9] that the same collapse of the vacillatory hierarchy occurs when returning to correct conjectures is disallowed. Thus, disallowing returning to wrong conjectures, disallowing returning to overinclusive conjectures and disallowing inverted-U-shapes each nullifies the extra power of finite vacillation with respect to convergence to a single correct conjecture. In contrast, we show that disallowing returning to overgeneralizing conjectures restricts the power of vacillatory learners in a less severe sense: for each $b>0$ there are classes of languages that are learnable with vacillation between at most $b+1$ correct conjectures in the limit by a learner not returning to old overgeneralizing conjectures, but not learnable by any learner who is allowed to vacillate between at most $b$ correct conjectures (Theorem 30). Also, we show that there are classes of languages that are learnable with vacillation between at most $b+1$ correct conjectures but such that any such learner must return to old overgeneralizing conjectures (Theorem 31). Hence if one disallows returning to old overgeneralizing conjectures on level 2 or above of the vacillatory learning hierarchy, then the power of the resulting criterion is weaker than the original one but still more powerful than explanatory learning.

In Section 6 we explore our four variants of non U-shaped learning in the context of TxtBc-learnability — when learners stabilize on (possibly infinite) sequences of grammars correctly describing the target language. First, 
we show that there exist TxtEx-learnable classes of languages that cannot be learned without returning to wrong conjectures even by TxtBc-learners. From this Theorem and results from [3], it follows that TxtBc-learners not returning to correct conjectures sometimes do better than those never returning to wrong conjectures. On the other hand, we show that, interestingly, TxtBc-learners not returning to wrong conjectures can sometimes do better than those never returning to correct conjectures. Therefore these two forms of non U-shaped behaviour (not returning to wrong conjectures and not returning to correct conjectures) are of incomparable strength in the context of TxtBc-learning. In contrast, we show that inverted U's are unnecessary in the context of TxtBc-learning (Theorem 44). The main result of this section is that, as in the case of TxtEx-learnability, returning to old overinclusive conjectures can be circumvented: every TxtBc-learner can be replaced by one not returning to overinclusive conjectures (Theorem 48). As a corollary, we obtain that returning to proper supersets of the target language is not necessary for full learning power in the TxtBc context (Corollary 49).

In Section 7 we discover a relationship between the strongest type of non Ushaped learners, that is decisive learners, and consistent learners [4,25], whose conjectures are required to be consistent with the input data seen so far. Consistent learnability is known to be weaker than general TxtEx-learnability $[4,25]$; moreover, sacrificing consistency, one can learn pattern languages faster than any consistent learner, under the assumption of $P \neq N P$ (see [22]). We show that consistent TxtEx-learners can be made consistent and decisive (Theorem 53). The result is surprising, since not returning to already used conjectures and being consistent with the input seen so far does not seem to be related - at least on the surface. On the other hand, some decisive learners cannot be made consistent (even if we sacrifice decisiveness).

In the concluding Section 8 we summarize our results, briefly consider their possible relevance from a cognitive science perspective, and formulate some open questions.

\section{Notation and Preliminaries}

Any unexplained recursion theoretic notation is from [28]. The symbol $\mathcal{N}$ denotes the set of natural numbers, $\{0,1,2,3, \ldots\}$. The symbols $\emptyset, \subseteq, \subset, \supseteq$, and $\supset$, denote empty set, subset, proper subset, superset and proper superset, respectively. The cardinality of a set $S$ is denoted by $\operatorname{card}(S) \cdot \operatorname{card}(S) \leq *$ denotes that $S$ is finite. The maximum and minimum of a set are denoted by $\max (\cdot), \min (\cdot)$, respectively, where $\max (\emptyset)=0$ and $\min (\emptyset)=\infty$.

We let $\langle\cdot, \cdot\rangle$ stand for Cantor's computable, bijective mapping $\langle x, y\rangle=\frac{1}{2}(x+y)$ $(x+y+1)+x$ from $\mathcal{N} \times \mathcal{N}$ onto $\mathcal{N}[28]$. Note that $\langle\cdot, \cdot\rangle$ is monotonically increasing in both of its arguments. We define $\pi_{1}(\langle x, y\rangle)=x$ and $\pi_{2}(\langle x, y\rangle)=$ $y$. 
Ву $\varphi$ we denote a fixed acceptable programming system for the partial-recursive functions mapping $\mathcal{N}$ to $\mathcal{N}[23,28]$. By $\varphi_{i}$ we denote the partial-recursive function computed by the program with number $i$ in the $\varphi$-system. The symbol $\mathcal{R}$ denotes the set of all recursive functions, that is total computable functions. By $\Phi$ we denote an arbitrary fixed Blum complexity measure $[6,19]$ for the $\varphi$-system. A partial recursive function $\Phi(\cdot, \cdot)$ is said to be a Blum complexity measure for $\varphi$, iff the following two conditions are satisfied:

(a) for all $i$ and $x, \Phi(i, x) \downarrow$ iff $\varphi_{i}(x) \downarrow$.

(b) the predicate: $P(i, x, t) \equiv \Phi(i, x) \leq t$ is decidable.

By convention we use $\Phi_{i}$ to denote the partial recursive function $x \rightarrow \Phi(i, x)$. Intuitively, $\Phi_{i}(x)$ may be thought as the number of steps it takes to compute $\varphi_{i}(x) . \varphi_{i, s}$ denotes the complexity-bounded version of $\varphi_{i}$, that is, $\varphi_{i, s}(x)=$ $\varphi_{i}(x)$, if $x<s$ and $\Phi_{i}(x)<s ; \varphi_{i, s}(x)$ is undefined otherwise.

By $W_{i}$ we denote domain $\left(\varphi_{i}\right)$. That is, $W_{i}$ is the set of all numbers on which the $\varphi$-program $i$ halts. Note that all acceptable numberings are isomorphic and thus one could also define $W_{i}$ to be the set generated by the $i$-th grammar. The symbol $\mathcal{E}$ will denote the set of all r.e. languages. The symbol $L$ ranges over $\mathcal{E}$. By $\bar{L}$, we denote the complement of $L$, that is $\mathcal{N}-L$. The symbol $\mathcal{L}$ ranges over subsets of $\mathcal{E}$. By $W_{i, s}$ we denote the set $\left\{x<s \mid \Phi_{i}(x)<s\right\}$.

We now present concepts from language learning theory. The next definition introduces the concept of a sequence of data.

Definition 1 (a) A sequence $\sigma$ is a mapping from an initial segment of $\mathcal{N}$ into $(\mathcal{N} \cup\{\#\})$. The empty sequence is denoted by $\lambda$.

(b) The content of a sequence $\sigma$, denoted content $(\sigma)$, is the set of natural numbers in the range of $\sigma$.

(c) The length of $\sigma$, denoted by $|\sigma|$, is the number of elements in $\sigma$. So, $|\lambda|=0$.

(d) For $n \leq|\sigma|$, the initial sequence of $\sigma$ of length $n$ is denoted by $\sigma[n]$. So, $\sigma[0]$ is $\lambda$.

Intuitively, the pause-symbol \# represents a pause in the presentation of data. We let $\sigma, \tau$ and $\gamma$ range over finite sequences. We denote the sequence formed by the concatenation of $\tau$ at the end of $\sigma$ by $\sigma \tau$. Sometimes we abuse the notation and use $\sigma x$ to denote the concatenation of sequence $\sigma$ and the sequence of length 1 which contains the element $x$. SEQ denotes the set of all finite sequences. We let $\delta_{0}, \delta_{1}, \ldots$ denote a standard recursive $1-1$ listing of all the finite sequences. We assume that $\max \left(\operatorname{content}\left(\delta_{i}\right)\right) \leq i$. We let $\operatorname{ind}(\sigma)$ denote $i$ such that $\delta_{i}=\sigma$.

We let $\operatorname{SEG}(L)$ denote the set $\{\sigma \mid \operatorname{content}(\sigma) \subseteq L\} . \sigma \preceq \tau(\sigma \prec \tau)$ denotes that $\sigma$ is an initial portion of (strict initial portion of) sequence $\tau$.

Definition 2 [18] (a) A text $T$ for a language $L$ is a mapping from $\mathcal{N}$ into $(\mathcal{N} \cup\{\#\})$ such that $L$ is the set of natural numbers in the range of $T . T(i)$ represents the $(i+1)$-st element in the text. 
(b) The content of a text $T$, denoted by content $(T)$, is the set of natural numbers in the range of $T$; that is, the language which $T$ is a text for.

(c) $T[n]$ denotes the finite initial sequence of $T$ with length $n$.

Definition 3 [18] A learning machine (or just learner) is an algorithmic device which computes a mapping from SEQ into $\mathcal{N}$.

We let $\mathbf{M}$ range over learning machines. We note that, without loss of generality, for all criteria of learning discussed in this paper, except for criteria involving consistent learning discussed in Section 7, a learner $\mathbf{M}$ may be assumed to be total. $\mathbf{M}(T[n])$ denotes the hypothesis of the learner $\mathbf{M}$ after it has seen the first $n$ members of $T$. It is expected that these hypotheses reflect more and more the nature of the set to be learned and this expectation will be made more formal in the following definitions.

There are several criteria for a learning machine to be successful on a language. Below we define some of them.

Definition 4 (Explanatory Learning, [12,18]) (a) M TxtEx-identifies a text $T$ just in case $\left(\exists i \mid W_{i}=\operatorname{content}(T)\right)\left(\forall^{\infty} n\right)[\mathbf{M}(T[n])=i]$.

(b) M TxtEx-identifies an r.e. language $L$ (written: $L \in \mathbf{T x t E x}(\mathbf{M})$ ) just in case M TxtEx-identifies each text for $L$.

(c) M TxtEx-identifies a class $\mathcal{L}$ of r.e. languages (written: $\mathcal{L} \subseteq \operatorname{TxtEx}(\mathbf{M})$ ) just in case $\mathbf{M}$ TxtEx-identifies each language from $\mathcal{L}$.

(d) TxtEx $=\{\mathcal{L} \subseteq \mathcal{E} \mid(\exists \mathbf{M})[\mathcal{L} \subseteq \operatorname{TxtEx}(\mathbf{M})]\}$.

Definition 5 (Behaviourally Correct Learning, [12])

(a) $\mathbf{M}$ TxtBc-identifies a text $T$ just in case $\left(\forall^{\infty} n\right)\left[W_{\mathbf{M}(T[n])}=\operatorname{content}(T)\right]$.

(b) $\mathbf{M} \mathbf{T x t B c}-i d e n t i f i e s$ an r.e. language $L$ (written: $L \in \mathbf{T x t B c}(\mathbf{M})$ ) just in case $\mathbf{M}$ TxtBc-identifies each text for $L$.

(c) M TxtBc-identifies a class $\mathcal{L}$ of r.e. languages (written: $\mathcal{L} \subseteq \operatorname{TxtBc}(\mathbf{M})$ ) just in case $\mathbf{M}$ TxtBc-identifies each language from $\mathcal{L}$.

(d) $\operatorname{TxtBc}=\{\mathcal{L} \subseteq \mathcal{E} \mid(\exists \mathbf{M})[\mathcal{L} \subseteq \operatorname{TxtBc}(\mathbf{M})]\}$.

Definition 6 (Vacillatory Learning, [11]) Suppose $a \in \mathcal{N} \cup\{*\}$.

(a) $\mathbf{M} \mathbf{T x t F e x}_{a}$-identifies a text $T$ just in case there exists a set $D, \operatorname{card}(D) \leq$ $a$ and $(\forall i \in D)\left[W_{i}=\operatorname{content}(T)\right]$, such that $\left(\forall^{\infty} n\right)\left[W_{\mathbf{M}(T[n])} \in D\right]$.

(b) $\mathbf{M} \mathbf{T x t F e x}_{a}$-identifies an r.e. language $L$ (written: $L \in \operatorname{TxtFex}_{a}(\mathbf{M})$ ) just in case $\mathbf{M} \mathbf{T x t F e x}_{a}$-identifies each text for $L$.

(c) $\mathbf{M}$ TxtFex $_{a}$-identifies a class $\mathcal{L} \subseteq \mathcal{E}$ (written: $\mathcal{L} \subseteq \operatorname{TxtFex}_{a}(\mathbf{M})$ ) just in case $\mathbf{M} \mathbf{T x t F e x}_{a}$-identifies each language from $\mathcal{L}$.

(d) $\operatorname{TxtFex}_{a}=\left\{\mathcal{L} \subseteq \mathcal{E} \mid(\exists \mathbf{M})\left[\mathcal{L} \subseteq \operatorname{TxtFex}_{a}(\mathbf{M})\right]\right\}$.

It is known that $\mathbf{T x t E x} \subset \mathbf{T x t F e x}_{2} \subset \mathbf{T x t F e x}_{3} \subset \ldots \subset \mathbf{T x t F e x}_{*} \subset \mathbf{T x t B c}$ (see [11-13]). 
Some of our proofs use the notion of stabilizing and locking sequences, as defined below.

Definition 7 (a) [16] $\sigma$ is said to be a stabilizing sequence for $\mathbf{M}$ on $L$ iff content $(\sigma) \subseteq L$, and for all $\tau \supseteq \sigma$ such that content $(\tau) \subseteq L, \mathbf{M}(\sigma)=\mathbf{M}(\tau)$.

(b) [5] $\sigma$ is said to be a $\mathbf{T x t E x}$-locking sequence for $\mathbf{M}$ on $L$ iff $\sigma$ is a stabilizing sequence for $\mathbf{M}$ on $L$, and $W_{\mathbf{M}(\sigma)}=L$.

(c) (Based on [5]) $\sigma$ is said to be a TxtBc-locking sequence for $\mathbf{M}$ on $L$ iff content $(\sigma) \subseteq L$, and for all $\tau \supseteq \sigma$ such that content $(\tau) \subseteq L, W_{\mathbf{M}(\sigma)}=L$.

(d) (Based on [5]) Let $b \in \mathcal{N} \cup\{*\} . \sigma$ is said to be a TxtFex $_{b}$-locking sequence for $\mathbf{M}$ on $L$ iff (i) content $(\sigma) \subseteq L$ and (ii) there exists a set $D$ of grammars for $L$, with $\operatorname{card}(D) \leq b$, such that $(\forall \tau \supseteq \sigma \mid \operatorname{content}(\tau) \subseteq L)[\mathbf{M}(\sigma) \in D]$.

Lemma 8 [5] If $\mathbf{M}$ TxtEx-identifies L, then there exists a TxtEx-locking sequence for $\mathbf{M}$ on $L$.

A similar result as the above can be proved for TxtBc- and for $\mathbf{T x t F e x}_{a^{-}}$ learning.

Let $\mathrm{INIT}_{k}$ denote the set $\{x \mid x \leq k\}$. Let INIT $=\left\{\operatorname{INIT}_{k} \mid k \in \mathcal{N}\right\}$.

\section{Decisive, Non U-Shaped and Related Criteria of Learning}

Firstly, we define the strongest type of non U-shaped behaviour — when a learner is not allowed to return to any old conjectures.

Definition 9 (Decisive Learner, [25]) (a) We say that $\mathbf{M}$ is decisive on text $T$, if there do not exist any $m, n, t$ such that $m<n<t, W_{\mathbf{M}(T[m])}=$ $W_{\mathbf{M}(T[t])}$ and $W_{\mathbf{M}(T[m])} \neq W_{\mathbf{M}(T[n])}$.

(b) We say that $\mathbf{M}$ is decisive on $L$ if $\mathbf{M}$ is decisive on each text for $L$.

(c) We say that $\mathbf{M}$ is decisive on $\mathcal{L}$ if $\mathbf{M}$ is decisive on each $L \in \mathcal{L}$.

Now we define non U-shaped learning.

Definition 10 (Non U-shaped Learner, [2]) (a) We say that $\mathbf{M}$ is non $U$ shaped on text $T$, if there do not exist any $m, n, t$ such that $m<n<t$, $W_{\mathbf{M}(T[m])}=W_{\mathbf{M}(T[t])}=\operatorname{content}(T)$ and $W_{\mathbf{M}(T[m])} \neq W_{\mathbf{M}(T[n])}$.

(b) We say that $\mathbf{M}$ is non $\mathbf{U}$-shaped on $L$ if $\mathbf{M}$ is non $\mathbf{U}$-shaped on each text for $L$.

(c) We say that $\mathbf{M}$ is non $\mathrm{U}$-shaped on $\mathcal{L}$ if $\mathbf{M}$ is non $\mathrm{U}$-shaped on each $L \in \mathcal{L}$.

Now we define our four models of non U-shaped learning when a learner is not allowed to return to previously used wrong conjectures ("Wr" in the next definition stands for "wrong").

Definition 11 (Wr-Decisive Learner) (a) We say that $\mathbf{M}$ is decisive on wrong conjectures (abbreviated $W r$-decisive) on text $T$, if there do not exist 
any $m, n, t$ such that $m<n<t, W_{\mathbf{M}(T[m])}=W_{\mathbf{M}(T[t])} \neq \operatorname{content}(T)$ and $W_{\mathbf{M}(T[m])} \neq W_{\mathbf{M}(T[n])}$.

(b) We say that $\mathbf{M}$ is Wr-decisive on $L$ if $\mathbf{M}$ is Wr-decisive on each text for $L$.

(c) We say that $\mathbf{M}$ is Wr-decisive on $\mathcal{L}$ if $\mathbf{M}$ is Wr-decisive on each $L \in \mathcal{L}$.

Now we define our model of non inverted-U-shaped learning.

Definition 12 (Non inverted-U-shaped Learner) (a) We say that $\mathbf{M}$ is non inverted- $U$-shaped on text $T$, if there do not exist any $m, n, t$ such that $m<n<t, W_{\mathbf{M}(T[m])}=W_{\mathbf{M}(T[t])} \neq W_{\mathbf{M}(T[n])}=\operatorname{content}(T)$.

(b) We say that $\mathbf{M}$ is non inverted-U-shaped on $L$ if $\mathbf{M}$ is non inverted-Ushaped on each text for $L$.

(c) We say that $\mathbf{M}$ is non inverted-U-shaped on $\mathcal{L}$ if $\mathbf{M}$ is non inverted-Ushaped on each $L \in \mathcal{L}$.

We now define our model of learning disallowing returning to conjectures containing elements outside the target language ("OI" in "OI-decisive" below stands for "overinclusive").

Definition 13 (OI-Decisive Learner) (a) We say that $\mathbf{M}$ is decisive on overinclusive conjectures (abbreviated OI-decisive) on text $T$, if there do not exist $m, n, t$ such that $m<n<t, W_{\mathbf{M}(T[m])}=W_{\mathbf{M}(T[t])} \nsubseteq \operatorname{content}(T)$ and $W_{\mathbf{M}(T[m])} \neq W_{\mathbf{M}(T[n])}$.

(b) We say that $\mathbf{M}$ is OI-decisive on $L$ if $\mathbf{M}$ is OI-decisive on each text for $L$.

(c) We say that $\mathbf{M}$ is OI-decisive on $\mathcal{L}$ if $\mathbf{M}$ is OI-decisive on each $L \in \mathcal{L}$.

We now introduce our model in which returning to proper supersets is disallowed ("OG" in "OG-decisive" below stands for "overgeneralizing").

Definition 14 (OG-Decisive Learner) (a) We say that $\mathbf{M}$ is decisive on overgeneralizing conjectures (abbreviated $O G$-decisive) on text $T$, if there do not exist $m, n, t$ such that $m<n<t, W_{\mathbf{M}(T[m])}=W_{\mathbf{M}(T[t])} \supset \operatorname{content}(T)$ and $W_{\mathbf{M}(T[m])} \neq W_{\mathbf{M}(T[n])}$.

(b) We say that $\mathbf{M}$ is OG-decisive on $L$ if $\mathbf{M}$ is OG-decisive on each text for $L$.

(c) We say that $\mathbf{M}$ is OG-decisive on $\mathcal{L}$ if $\mathbf{M}$ is OG-decisive on each $L \in \mathcal{L}$.

We now define the learning criteria formed by placing the various constraints described above on the learner. Note that the definition used for decisive learning is the class version of decisive, that is, decisiveness is required to hold only for texts for the languages in the class. We do this to make it consistent with the definitions of non $U$-shaped, Wr-decisive, non inverted$U$-shaped, OI-decisive and OG-decisive criteria, where only the class version seems sensible.

Definition 15 (a) [25] We say that M DecEx-identifies $L$ (written: $L \in$ $\operatorname{DecEx}(\mathbf{M})$ ), iff $\mathbf{M} \mathbf{T x t E x}$-identifies $L$, and $\mathbf{M}$ is decisive on $L$.

We say that $\mathbf{M}$ DecEx-identifies $\mathcal{L}$, iff $\mathbf{M}$ DecEx-identifies each $L \in \mathcal{L}$.

$\operatorname{DecEx}=\{\mathcal{L} \mid(\exists \mathbf{M})[\mathcal{L} \subseteq \operatorname{DecEx}(\mathbf{M})]\}$. 
(b) [2] We say that $\mathbf{M} \mathbf{N U S h E x}$-identifies $L$ (written: $L \in \mathbf{N U S h E x}(\mathbf{M})$ ), iff $\mathbf{M}$ TxtEx-identifies $L$, and $\mathbf{M}$ is non $\mathrm{U}$-shaped on $L$.

We say that $\mathbf{M}$ NUShEx-identifies $\mathcal{L}$, iff $\mathbf{M}$ NUShEx-identifies each $L \in \mathcal{L}$.

$\operatorname{NUShEx}=\{\mathcal{L} \mid(\exists \mathbf{M})[\mathcal{L} \subseteq \operatorname{NUShEx}(\mathbf{M})]\}$.

(c) We say that $\mathbf{M} \mathbf{W r D E x}$-identifies $L$ (written: $L \in \mathbf{W r D E x}(\mathbf{M})$ ), iff $\mathbf{M}$ TxtEx-identifies $L$, and $\mathbf{M}$ is $\mathrm{Wr}$-decisive on $L$.

We say that $\mathbf{M} \mathbf{W r D E x}$-identifies $\mathcal{L}$, iff $\mathbf{M} \mathbf{W r D E x}$-identifies each $L \in \mathcal{L}$.

$\operatorname{WrDEx}=\{\mathcal{L} \mid(\exists \mathbf{M})[\mathcal{L} \subseteq \operatorname{WrDEx}(\mathbf{M})]\}$.

(d) We say that $\mathbf{M}$ NInvUEx-identifies $L$ (written: $L \in \mathbf{N I n v} \mathbf{U E x}(\mathbf{M})$ ), iff M TxtEx-identifies $L$, and $\mathbf{M}$ is non inverted-U-shaped on $L$.

We say that $\mathbf{M}$ NInvUEx-identifies $\mathcal{L}$, iff $\mathbf{M}$ NInvUEx-identifies each $L \in \mathcal{L}$.

NInvUEx $=\{\mathcal{L} \mid(\exists \mathbf{M})[\mathcal{L} \subseteq \operatorname{NInv} \mathbf{U E x}(\mathbf{M})]\}$.

(e) We say that $\mathbf{M}$ OIDEx-identifies $L$ (written: $L \in \operatorname{OIDEx}(\mathbf{M})$ ), iff $\mathbf{M}$ TxtEx-identifies $L$, and $\mathbf{M}$ is OI-decisive on $L$.

We say that $\mathbf{M}$ OIDEx-identifies $\mathcal{L}$, iff $\mathbf{M}$ OIDEx-identifies each $L \in \mathcal{L}$.

OIDEx $=\{\mathcal{L} \mid(\exists \mathbf{M})[\mathcal{L} \subseteq \operatorname{OIDEx}(\mathbf{M})]\}$.

(f) We say that $\mathbf{M}$ OGDEx-identifies $L$ (written: $L \in \mathbf{O G D E x}(\mathbf{M})$ ), iff $\mathbf{M}$ TxtEx-identifies $L$, and $\mathbf{M}$ is OG-decisive on $L$.

We say that M OGDEx-identifies $\mathcal{L}$, iff $\mathbf{M}$ OGDEx-identifies each $L \in \mathcal{L}$.

$\operatorname{OGDEx}=\{\mathcal{L} \mid(\exists \mathbf{M})[\mathcal{L} \subseteq \operatorname{OGDEx}(\mathbf{M})]\}$.

One can similarly define DecJ, WrDJ, NInvUJ, OIDJ, OGDJ and NUShJ, for $\mathbf{J} \in\left\{\mathbf{F e x}_{a}, \mathbf{B c}\right\}$.

The following result is easy to verify.

Proposition 16 Suppose $a \in \mathcal{N} \cup\{*\}$ and $\mathbf{J} \in\left\{\mathbf{E x}, \mathbf{F e x}_{a}, \mathbf{B c}\right\}$.

(a) DecJ $\subseteq \mathbf{W r D J} \subseteq$ OIDJ $\subseteq$ OGDJ $\subseteq \mathbf{J}$.

(b) DecJ $\subseteq \mathbf{W r D J} \subseteq \mathbf{N I n v} \mathbf{U J} \subseteq \mathbf{J}$.

(c) DecJ $\subseteq$ NUShJ $\subseteq$ NInvUJ $\subseteq$ J.

For our proofs, we will be using the following results from [3].

Lemma 17 [3] Suppose $\mathbf{M} \mathbf{T x t E x}$-identifies $\mathcal{L}$, and $g$ is a recursive function such that

(I) $W_{g(i)} \neq W_{g(j)}$, for $i \neq j$,

(II) for all finite sets $S$, there exist infinitely many $i$ such that $S \subseteq W_{g(i)}$,

(III) $W_{\mathbf{M}(\sigma)} \notin\left\{W_{g(i)} \mid i \in \mathcal{N}\right\}$, for all $\sigma$.

Then, $\mathcal{L} \in$ DecEx.

Proposition 18 [3] Suppose $\mathcal{L} \in \mathbf{T x t E x}$ and $\mathcal{N} \in \mathcal{L}$. Then, $\mathcal{L} \in$ DecEx. 


\section{Explanatory Learning}

Our first goal is to show that, in the context of TxtEx-learnability, learners not returning to wrong conjectures do no better than decisive learners. To prove this, we first establish two lemmas.

Lemma 19 Suppose there exists a finite set $A$ such that $\mathcal{L}$ does not contain any extension of $A$. Then $\mathcal{L} \in \mathbf{T x t E x} \Rightarrow \mathcal{L} \in \mathbf{D e c E x}$.

Proof. Suppose $\mathcal{L} \subseteq \operatorname{TxtEx}(\mathbf{M})$. Without loss of generality suppose $\mathbf{M}$ never outputs an extension of $A$ : This can be achieved by converting any grammar $i$ to $f(i)$ where

$$
W_{f(i)}=\bigcup_{s \in \mathcal{N}} X_{s} \text { and } X_{s}= \begin{cases}W_{i, s}, & \text { if } A \nsubseteq W_{i, s} \\ \emptyset, & \text { otherwise. }\end{cases}
$$

Clearly, $W_{f(i)}$ does not contain $A$. Furthermore, $W_{i}=W_{f(i)}$, if $A \nsubseteq W_{i}$.

Let $m=\max (A)$, and let $W_{g(i)}=\{0,1, \ldots, m+i\} \cup\{m+i+2\}$, so that $W_{g(i)}$ is an extension of $A$ and is not an initial segment of $\mathcal{N} . W_{g(i)}$ are pairwise distinct and every finite set is extended by infinitely many $W_{g(i)}$ 's. Furthermore, $\left\{W_{g(i)} \mid i \in \mathcal{N}\right\}$ is disjoint from $\left\{W_{\mathbf{M}(\sigma)} \mid \sigma \in \mathrm{SEQ}\right\}$. Therefore by Lemma 17, $\mathcal{L} \in$ DecEx.

Lemma 20 Suppose every finite set has at least two extensions in $\mathcal{L}$. Suppose $a \in \mathcal{N} \cup\{*\}$ and $\mathbf{J} \in\left\{\mathbf{E x}, \mathbf{F e x}_{a}, \mathbf{B c}\right\}$. Then, $\mathcal{L} \subseteq \operatorname{DecJ}(\mathbf{M})$ iff $\mathcal{L} \subseteq$ $\operatorname{WrDJ}(\mathbf{M})$.

Proof. Suppose by way of contradiction that $\mathcal{L} \subseteq \operatorname{WrDJ}(\mathbf{M}), \mathcal{L} \nsubseteq \operatorname{DecJ}(\mathbf{M})$. Thus, $\mathbf{M}$ is not decisive. Let $\tau_{1} \prec \tau_{2} \prec \tau_{3}$ be such that $W_{\mathbf{M}\left(\tau_{1}\right)}=W_{\mathbf{M}\left(\tau_{3}\right)} \neq$ $W_{\mathbf{M}\left(\tau_{2}\right)}$. Let $L$ be an extension of $\operatorname{content}\left(\tau_{3}\right)$ such that $W_{\mathbf{M}\left(\tau_{1}\right)} \neq L$ and $L \in \mathcal{L}$. Such an $L$ exists by the assumptions on $\mathcal{L}$. Let $T$ be a text for $L$ extending $\tau_{3}$. Then $T$ witnesses that $\mathbf{M}$ does not $\mathbf{W r D J}$-identify $\mathcal{L}$ since $\mathbf{M}$ returns to the wrong conjecture $W_{\mathbf{M}\left(\tau_{1}\right)}$ on text $T$. A contradiction. The Lemma is thus proved.

Now we can establish one of our main results.

Theorem 21 DecEx $=$ WrDEx.

Proof. Suppose $\mathcal{L} \in \mathbf{W r D E x}$. We consider the following cases.

Case 1: $\mathcal{L}$ contains at least two extensions of every finite set. Then by Lemma $20, \mathcal{L}$ is in DecEx.

Case 2: Not Case 1 , and $\mathcal{N} \in \mathcal{L}$. Then by Proposition 18, we have that $\mathcal{L} \in$ DecEx.

Case 3: Neither Case 1 nor Case 2.

Since Case 1 does not hold, there is a finite set $A$ such that $\mathcal{L}$ contains at most one extension of $A$. If such an extension $L$ of $A$ exists, then $L \neq \mathcal{N}$ and thus there is an element $w \notin L$. If such an extension $L$ of $A$ does not exist then let 
$w=0$. Now $\mathcal{L}$ does not contain any superset of $A \cup\{w\}$ and $\mathcal{L} \in$ DecEx by Lemma 19.

As DecEx $\subset$ TxtEx [3], we conclude that some families of languages in TxtEx cannot be learned without returning to wrong conjectures.

However, if we allow returning to subsets of the target language (that is, wrong conjectures that are not overinclusive), then all classes of languages in TxtEx become learnable, as the following result shows.

\section{Theorem 22 TxtEx $\subseteq$ OIDEx.}

Proof. Suppose $\mathcal{L} \in \mathrm{TxtEx}$.

If $\mathcal{N} \in \mathcal{L}$, then by Proposition $18, \mathcal{L} \in$ DecEx. Thus, by Proposition 16, $\mathcal{L} \in$ OIDEx. So assume $\mathcal{N} \notin \mathcal{L}$. Let $\mathbf{M}$ be a machine such that, (I) $\mathbf{M}$ TxtEx-identifies $\mathcal{L} \cup$ INIT, and (II) all texts for $L \in \mathcal{L} \cup$ INIT, start with a TxtEx-locking sequence for $\mathbf{M}$ on $L$, and (III) for all $k$, if $\sigma$ is a stabilizing sequence for $\mathbf{M}$ on $I N I T_{k}$, then $\operatorname{content}(\sigma)=I N I T_{k}$.

Note that Fulk [16] shows that this can be assumed without loss of generality when $\mathcal{N} \notin \mathcal{L}$, (where property (III) above can be obtained by slight modification of his proof - by assuming that special indices for INIT are used when the content of input segment is for a member of INIT).

Below we will define $\mathbf{M}^{\prime}$ which OIDEx-identifies $\mathcal{L}$. Intuitively, the idea of the proof is to basically follow conjectures of $\mathbf{M}$, if the relevant initial segment $T[n]$ of input text $T$ (on which the conjecture is based) seems to be a locking sequence for the conjecture; otherwise we choose an appropriate member of INIT to be the conjecture of $\mathbf{M}^{\prime}$, which allows us to preserve non-repetition of over-inclusive conjectures. We now proceed formally.

For a segment $\sigma$, let $f(\sigma)=\min (\mathcal{N}-\operatorname{content}(\sigma))$. We say that $T[m]$ is valid if $m=0$ or $\mathbf{M}(T[m-1]) \neq \mathbf{M}(T[m])$. Let consseq $=\{T[m] \mid \operatorname{content}(T[m]) \subseteq$ $\left.W_{\mathbf{M}(T[m])}\right\}$. Let gram be a recursive function such that

$$
W_{\operatorname{gram}(T[m])}=\left\{\begin{array}{cc}
\emptyset, & \text { if } \operatorname{content}(T[m]) \nsubseteq W_{\mathbf{M}(T[m])} ; \\
W_{\mathbf{M}(T[m])}, & \text { if } T[m] \text { is a stabilizing sequence for } W_{\mathbf{M}(T[m])} ; \\
I N I T_{\langle\text {ind }(T[m]), w\rangle}, & \text { otherwise, for some } w \geq f(T[m]) .
\end{array}\right.
$$

It is easy to verify that for $T[m] \in$ consseq, content $(T[m]) \subseteq W_{\text {gram }(T[m])}$ (in the second clause, this follows by definition of stabilizing sequence; in the third clause, this follows as $\operatorname{ind}(T[m]) \geq \max (\operatorname{content}(T[m]))$, by the definition of indexing of finite sequences considered in Section 2, and the monotonicity of pairing function).

Define $\mathbf{M}^{\prime}$ as follows. $\mathbf{M}^{\prime}(T[n])=\operatorname{gram}(T[m])$, for the largest $m \leq n$, such that $T[m]$ is valid and $W_{\mathbf{M}(T[m]), n} \supseteq \operatorname{content}(T[m])$ (there exists such an $m$, 
as $m=0$ satisfies the constraints). Note that the mapping from $n$ to that $m$ for which $\mathbf{M}^{\prime}(T[n])=\operatorname{gram}(T[m])$, is monotonically non-decreasing in $n$ on its domain.

It is easy to verify that $\mathbf{M}^{\prime} \mathbf{T x t E x}$-identifies $\mathcal{L}$ using the assumptions (I), (II) and (III) about $\mathbf{M}$. Thus, it remains to show that $\mathbf{M}^{\prime}$ is OI-decisive.

Suppose $T$ is a text for $L \in \mathcal{L}$. We now show that if $W_{\mathbf{M}^{\prime}\left(T\left[m^{\prime}\right]\right)}=W_{\mathbf{M}^{\prime}\left(T\left[n^{\prime}\right]\right)} \neq$ $W_{\mathbf{M}^{\prime}\left(T\left[s^{\prime}\right]\right)}$, for $m^{\prime}<s^{\prime}<n^{\prime}$, then $W_{\mathbf{M}^{\prime}\left(T\left[m^{\prime}\right]\right)} \subseteq L$.

So suppose $m^{\prime}, s^{\prime}, n^{\prime}$ as above are given. Suppose $\mathbf{M}^{\prime}\left(T\left[m^{\prime}\right]\right)=\operatorname{gram}(T[m])$, $\mathbf{M}^{\prime}\left(T\left[s^{\prime}\right]\right)=\operatorname{gram}(T[s])$, and $\mathbf{M}^{\prime}\left(T\left[n^{\prime}\right]\right)=\operatorname{gram}(T[n])$. By monotonicity property of $\mathbf{M}^{\prime}$ mentioned above, $m^{\prime}<s^{\prime}<n^{\prime}$ implies $m \leq s \leq n$. If $m=n$, then it yields a contradiction, as $\mathbf{M}^{\prime}\left(T\left[s^{\prime}\right]\right)$ would also be equal to $\operatorname{gram}(T[m])$. Thus, $m<n$. As $T[n]$ is valid and $\operatorname{content}(T[n]) \subseteq W_{\text {gram }(T[n])}$ (by remark just after the definition of gram), we immediately have that $T[\mathrm{~m}]$ is not a stabilizing sequence for $\mathbf{M}$ on $W_{\mathbf{M}(T[m])}=W_{\mathbf{M}(T[n])} \supseteq \operatorname{content}(T[n])$. Thus, $\operatorname{gram}(T[m])$ follows the third clause in the definition of $\operatorname{gram}$. Since, $\langle\operatorname{ind}(T[m]), \cdot\rangle \neq$ $\langle\operatorname{ind}(T[n]), \cdot\rangle$, for $m \neq n$, it follows that $\operatorname{gram}(T[n])$ must follow the second clause, and thus $T[n]$ is a stabilizing sequence for $W_{\mathbf{M}(T[n])}$. As $W_{\text {gram }(T[m])}$ $\left(=W_{\operatorname{gram}(T[n])}\right)$ is in INIT, it follows that content $(T[n])=W_{\operatorname{gram}(T[n])}($ since $\sigma$ being stabilizing sequence for $\mathbf{M}$ on $I N I T_{k}$ implies that content $\left.(\sigma)=I N I T_{k}\right)$. Thus, $W_{\operatorname{gram}(T[m])}=W_{\operatorname{gram}(T[n])}=\operatorname{content}(T[n]) \subseteq L$.

It follows that $\mathbf{M}^{\prime}$ OIDEx-identifies $\mathcal{L}$.

By definition of OGDEx we have the following Corollary.

\section{Corollary 23 TxtEx $\subseteq$ OGDEx.}

Recall the following result about non U-shaped learners from [3].

Theorem $24[3]$ (a) TxtEx $\nsubseteq$ DecBc.

(b) TxtEx = NUShEx.

Clearly, NUShEx $\subseteq$ NInvUEx $\subseteq$ TxtEx. Thus from Theorem 24 we have the following.

\section{Corollary 25 NInvUEx = TxtEx.}

Theorems 24 and 21 imply that disallowing returning to abandoned wrong conjectures is more restrictive than disallowing returning to abandoned correct conjectures in the context of TxtEx-learning. From Theorem 22, Corollaries 23 and 25, the latter requirement of disallowing returning to abandoned correct conjectures is equivalent to disallowing inverted U's and to disallowing returning to abandoned overinclusive or to overgeneralizing conjectures. We summarize these observations in the following immediate corollary.

Corollary 26 (a) WrDEx $\subset$ NUShEx.

(b) NUShEx $=$ OIDEx $=$ OGDEx $=$ NInvUEx. 


\section{$5 \quad$ Vacillatory Learning}

In this section we show that when returning to wrong conjectures is not allowed in vacillatory learning, then the vacillatory hierarchy $\mathbf{T x t F e x}_{1} \subset \mathbf{T x t F e x}_{2} \subset$ $\ldots \subset \mathbf{T x t F e x}_{*}$ collapses to $\mathbf{T x t F e x}_{1}=\mathbf{T x t E x}$, so that the extra learning power given by vacillation is lost. That the same collapse occurs when returning to correct abandoned conjectures is disallowed was shown in [9].

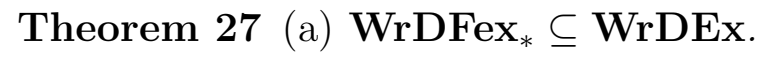

(b) NInvUFex $_{*} \subseteq$ TxtEx.

(c) $\mathrm{OIDFex}_{*} \subseteq$ TxtEx.

Proof. (a) Suppose $\mathbf{M} \mathbf{W r D F e x}_{*}$-identifies $\mathcal{L}, L \in \mathcal{L}$ and $T$ is a text for the language $L$.

Let us define a symmetric relation $E_{a}$ as follows: $E_{a}(i, j)$ holds iff there exist $n_{1}, n_{2}, n_{3}, n_{4}$ such that $n_{1}<n_{2}<n_{3}<n_{4} \leq a, \mathbf{M}\left(T\left[n_{1}\right]\right)=\mathbf{M}\left(T\left[n_{3}\right]\right)$, $\mathbf{M}\left(T\left[n_{2}\right]\right)=\mathbf{M}\left(T\left[n_{4}\right]\right)$ and $\left\{\mathbf{M}\left(T\left[n_{1}\right]\right), \mathbf{M}\left(T\left[n_{2}\right]\right)\right\}=\{i, j\}$ where $a \in\{0,1,2$, $\ldots, *\}$. That is, $E_{a}(i, j)$ holds if the learner alternates at least three times between these two hypotheses with possibly other hypotheses conjectured in between and this alternation occurs on an initial segment of length up to $a$; this last restriction on the length of the initial segment is void for $a=*$.

Note that $E_{a}(i, j)$ implies $W_{i}=W_{j}$ : assuming by way of contradiction that $W_{i} \neq W_{j}$, the learner would return to the abandoned hypotheses $W_{i}$ and $W_{j}$; by definition of $\mathbf{W r D F e x}_{*}$, each of these hypothesis could not be wrong; thus both would have to be correct, and hence equal, contrary to the assumption.

By taking reflexive and transitive closure of $E_{a}$, we get an equivalence relation $\tilde{E}_{a}$. Note that still $W_{i}=W_{j}$ whenever $\tilde{E}_{a}(i, j)$.

Now a new learner $\mathbf{M}^{\prime}$ is built by defining $\mathbf{M}^{\prime}(T[n])$ to be a canonical index for the union of those $W_{e}$ for which $e$ satisfies $\tilde{E}_{n}(e, \mathbf{M}(T[n]))$.

First note that $\mathbf{M}^{\prime}$ is well-defined since there are only finitely many such $e$ with $\tilde{E}_{n}(e, \mathbf{M}(T[n]))$. Each such $e$ has to be of the form $\mathbf{M}(T[m])$ for some $m \leq n$.

Second, $W_{\mathbf{M}^{\prime}(T[n])}=W_{\mathbf{M}(T[n])}$ for all $n$. To see this note that $e=\mathbf{M}(T[n])$ satisfies $\tilde{E}_{n}(e, \mathbf{M}(T[n]))$ and thus the union is over a nonempty class of sets. Furthermore, all the sets in this class are equal since $\tilde{E}_{n}(e, \mathbf{M}(T[n]))$ implies $W_{e}=W_{\mathbf{M}(T[n])}$. So the union of the sets $W_{e}$ with $\tilde{E}_{n}(e, \mathbf{M}(T[n]))$ is the set $W_{\mathbf{M}(T[n])}$.

Third, as $\mathbf{M}$ WrDBc-identifies $L$ from $T$ so does $\mathbf{M}^{\prime}$.

Fourth, it is easy to verify that all grammars $i$, which are output infinitely often by $\mathbf{M}$ on text $T$, belong to the same $\tilde{E}_{*}$-equivalence class $D$. Since, $\mathbf{M}$ $\mathbf{W r D F e x}_{*}$-identifies $L$, this set $D$ is finite. Also, there is an $m$ such that for all $n \geq m$, for all $i, j \in D, \tilde{E}_{n}(i, j)$. Thus $\mathbf{M}^{\prime}(T[n])$ is the same index for the union 
of the $W_{e}$, with $e \in D$, whenever $n \geq m$ and $\mathbf{M}(T[n]) \in D$. As $\mathbf{M} \mathbf{T x t F e x}_{*^{-}}$ identifies $L, \mathbf{M}(T[n]) \in D$ for almost all $n$. Thus, $\mathbf{M}^{\prime}$ even $\mathbf{W r D E x}$-identifies $L$ from $T$.

In particular, $\mathbf{M}^{\prime}$ is a $\mathbf{W r D E x}$-identifier for $\mathcal{L}$.

(b) The proof is analogue to (a) with the following differences: The relation $\tilde{E}_{a}(i, j)$ does not imply that $W_{i}=W_{j}$ but just that $W_{i}=L \Leftrightarrow W_{j}=L$. So $D$ contains only correct indices and $\mathbf{M}^{\prime}$ is a $\mathbf{T x t E x}$-identifier for $\mathcal{L}$, but it is not guaranteed that the NInvU-property is preserved. In particular the second item of the verification breaks down, but the third and fourth items can make use of the fact that all members of $D$ are correct indices and thus the union of the sets with indices in $D$ is the set $L$ to be learned.

(c) This part is similar to part (b), except that in this case, we have that $E_{a}(i, j)$ implies $W_{i} \subseteq L \Leftrightarrow W_{j} \subseteq L$. This follows from the definition of OIDFex-identification as either $W_{i}=W_{j}$ or both are subsets of the input language. Finally all indices which are output infinitely often are correct, so $D$ contains at least one correct index and perhaps some additional indices of subsets of $L$. So the union of the sets with indices in $D$ gives the set $L$.

Since every explanatory learner is by definition also a vacillatory learner, the inclusion (a) of the previous Theorem is not proper. Furthermore, using Theorem 21 from the previous section, we actually get decisiveness on the right side of the equality. Furthermore, the second and third inclusion of the previous Theorem can be improved by using the equalities OIDEx $=$ NInvUEx = TxtEx (see Theorems 22 and 24).

Corollary 28 (a) $\mathrm{WrDFex}_{*}=$ DecEx

(b) $\mathrm{OIDFex}_{*}=$ OIDEx.

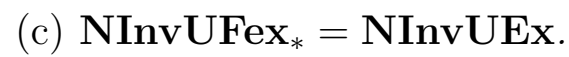

From the above Corollary we can conclude that, as was the case for TxtExlearning, WrD is more restrictive than NUSh while NInvU and OID are equivalent to NUSh.

A subtler difference between returning to wrong conjectures and returning to correct conjectures in the context of vacillatory learning can be observed. Recall the following result from [9].

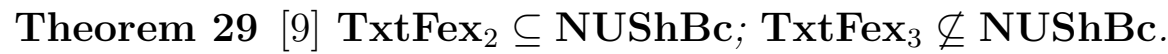

Thus, returning to correct conjectures is avoidable for the $\mathbf{T x t F e x}_{2}$ level of the vacillatory hierarchy by shifting to the more liberal criterion of TxtBc identification, while this is not the case for $\mathbf{T x t F e x}_{b}$ with $b \geq 3$. In the next section we prove (Theorem 34) that there are TxtEx-learnable classes that cannot be TxtBc-learned by any WrD-learner ${ }^{4}$. Thus, the necessity of returning to wrong abandoned conjectures is not avoidable by allowing infinitely

$\overline{4}$ Observe that this result is not a trivial consequence of TxtEx $\not \subset$ DecBc from [3], since we show in the next section (Corollary 39) that DecBc $\subset \mathbf{W r D B c}$. 
many correct grammars in the limit, not even for the $\mathbf{T x t F e x}_{2}$ level of the vacillatory hierarchy. In this sense, we can say that the necessity of returning to wrong conjectures is even deeper than the necessity of returning to correct conjectures.

We now show that disallowing returning to old overgeneralizing conjectures still restricts the full learning power of vacillatory learning, but in a different and less severe way. First we show that, for each $n>0$, there are classes that are OGD-learnable with vacillation between at most $n+1$ correct conjectures but not learnable at all with vacillation between at most $n$ conjectures. Thus the vacillatory hierarchy does not collapse when returning to overgeneralizing hypotheses is disallowed.

Theorem 30 For $n>0$, OGDFex ${ }_{n+1} \nsubseteq$ TxtFex $_{n}$.

Proof. $D_{0}, D_{1}, \ldots$, denotes a canonical recursive indexing of all the finite sets [28, Page 70], such that the elements and the size of $D_{j}$ can be effectively determined from $j$. For each $j$ let $X_{j}=\{\langle j, x\rangle \mid x \in \mathcal{N}\}$. Let $\mathcal{L}=\{L \mid$ $(\exists j)\left[\emptyset \subset L \subseteq X_{j}\right.$ and $\operatorname{card}\left(D_{j}\right) \leq n+1$ and $\left(\exists p \in D_{j}\right)\left[L=W_{p}\right]$ and $(\forall k \in$ $\left.\left.\left.D_{j}\right)\left[L \not \subset W_{k}\right]\right]\right\}$.

Clearly, $\mathcal{L} \in$ OGDFex $_{n+1}$, as, on input $\sigma$ with non-empty content, a learner can first obtain a $j$ such that $L \subseteq X_{j}$, and then output the $p \in D_{j}$ which maximizes $\left|\tau_{p}\right|$, where $\tau_{p}$ is the maximal prefix of $\sigma$ such that $\operatorname{content}\left(\tau_{p}\right) \subseteq$ $W_{p,|\sigma|}$. If $\operatorname{content}(\sigma)=\emptyset$, then the learner outputs a hypothesis for $\emptyset$. This learner clearly OGDFex $_{n+1}$-identifies $\mathcal{L}$.

The diagonalization proof is essentially based on the proof of $\operatorname{TxtFex}_{n+1} \nsubseteq$ TxtFex $_{n}$ from [11]. Suppose by way of contradiction that M TxtFex ${ }_{n}$-identifies $\mathcal{L}$. Then, by $(n+1)$-ary recursion theorem [28] there exist distinct $e_{1}, \ldots, e_{n+1}$ such that $W_{e_{1}}, \ldots, W_{e_{n+1}}$ may be defined as follows. Let $j$ be such that $D_{j}=\left\{e_{1}, \ldots, e_{n+1}\right\}$. Initially let $\sigma_{0}$ be such that content $\left(\sigma_{0}\right)=\{\langle j, 0\rangle\}$, and enumerate $\langle j, 0\rangle$ in each of $W_{e_{i}}, 1 \leq i \leq n+1$. For any sequence $\sigma$, let Last $_{n}(\sigma)$ denote the set of the last $n$ grammars output by $\mathbf{M}$ on input $\sigma$. That is $\operatorname{Last}_{n}(\sigma)=\left\{\mathbf{M}(\tau) \mid \tau \preceq \sigma \wedge \operatorname{card}\left(\left\{\mathbf{M}\left(\tau^{\prime}\right) \mid \tau \preceq \tau^{\prime} \preceq \sigma\right\}\right) \leq n\right\}$. Go to stage 0 .

Stage $s$

1. Dovetail steps 2 and 3 until, if ever, step 2 succeeds. If and when step 2 succeeds, stop step 3 and go to step 4 .

2. Search for an extension $\tau$ of $\sigma_{s}$ such that content $(\tau) \subseteq X_{j}$ and $\operatorname{Last}_{n}(\tau) \neq$ $\operatorname{Last}_{n}\left(\sigma_{s}\right)$.

3. For $r=1$ to $\infty$ Do

Begin For $k=1$ to $n+1$, enumerate $\langle j,\langle k, r\rangle\rangle$ into $W_{e_{k}}$ End.

4. Let $S$ be the set of all the elements enumerated into $W_{e_{1}} \cup W_{e_{2}} \cup \ldots W_{e_{k}}$ up to now.

Let $\sigma_{s+1}$ be an extension of $\tau$ such that $\operatorname{content}\left(\sigma_{s+1}\right)=\operatorname{content}(\tau) \cup S$.

For $k=1$ to $n+1$, enumerate $\operatorname{content}\left(\sigma_{s+1}\right)$ into $W_{e_{k}}$. 
Go to stage $s+1$.

End stage $s$.

We now consider the following cases.

Case 1: There exist infinitely many stages.

In this case, clearly, $W_{e_{1}}=W_{e_{2}}=\ldots=W_{e_{n+1}}$, and $\mathbf{M}$ does not converge to a set of $n$ grammars on $\bigcup_{s \in \mathcal{N}} \sigma_{s}$, a text for $L=W_{e_{1}}$, which is a member of $\mathcal{L}$.

Case 2: Stage $s$ starts but never ends.

In this case consider $L_{k}=W_{e_{k}}$, for $1 \leq k \leq n+1$. Note that $L_{k} \subseteq X_{j}$ and for $1 \leq k, k^{\prime} \leq n+1, k \neq k^{\prime}: L_{k} \nsubseteq L_{k^{\prime}}$. Thus, each $L_{k}$ belongs to $\mathcal{L}$. Furthermore for $1 \leq k \leq n+1$, for any text $T$ for $L_{k}$ which extends $\sigma_{s}$, all grammars which are output by $\mathbf{M}$ on $T$ beyond $\sigma_{s}$, are from $\operatorname{Last}_{n}\left(\sigma_{s}\right)$ (otherwise step 2 would succeed as $L_{k} \subseteq X_{j}$ ). Thus, $\mathbf{M}$ fails to $\mathbf{T x t F e x}_{n}$-identify at least one of $L_{k}, 1 \leq k \leq n+1$ (since Last $_{n}\left(\sigma_{s}\right)$ can contain grammars for at most $n$ of $\left.L_{1}, \ldots, L_{n+1}\right)$.

It follows from above cases that $\mathbf{M}$ cannot $\mathbf{T x t F e x}_{n}$-identify $\mathcal{L}$.

Next we show that the learning power of each level of the vacillatory hierarchy is restricted when returning to overgeneralizing conjectures is disallowed. More precisely, there are classes that are learnable with vacillation between two correct indices in the limit but such that no vacillatory learner can learn those classes without returning to overgeneralizing conjectures, no matter what amount of vacillation is allowed.

Theorem 31 TxtFex $_{2} \nsubseteq$ OGDFex $_{*}$.

Proof. Let $L_{i}=\{\langle i, x\rangle \mid x \in \mathcal{N}\}$. Let $S_{i}=\left\{\langle i, x\rangle \mid x \leq \operatorname{card}\left(W_{i}\right)\right\}$ (thus, if $\operatorname{card}\left(W_{i}\right)=\infty$, then $\left.S_{i}=L_{i}\right)$.

Let $\mathcal{L}=\left\{L_{i} \mid i \in \mathcal{N}\right\} \cup\left\{S_{i} \mid i \in \mathcal{N}\right\}$. It is easy to verify that $\mathcal{L} \in \mathbf{T x t F e x}_{2}$.

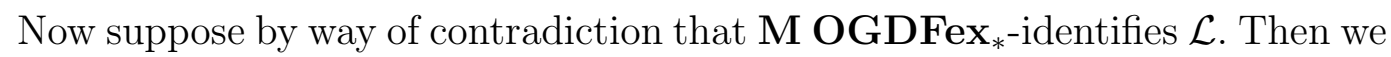
show that $\operatorname{In} f=\left\{i \mid \operatorname{card}\left(W_{i}\right)=\infty\right\}$ is $\Sigma_{2}$, a contradiction to $\Pi_{2}$ completeness of Inf (see [28] for definition of $\Sigma_{2}, \Pi_{2}$ and $\Pi_{2}$ completeness of $\mathrm{Inf}$ ).

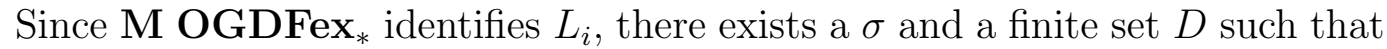
(I) $\sigma \in \operatorname{SEG}\left(L_{i}\right),($ II $)\left(\forall j, j^{\prime} \in D\right)\left(\exists \tau_{1}, \tau_{2}, \tau_{3}\right)\left[\tau_{1} \preceq \tau_{2} \preceq \tau_{3} \preceq \sigma\right.$ and $\mathbf{M}\left(\tau_{1}\right)=$ $\left.j, \mathbf{M}\left(\tau_{2}\right)=j^{\prime}, \mathbf{M}\left(\tau_{3}\right)=j\right]$ and $(\mathrm{III})\left(\forall \tau \in \operatorname{SEG}\left(L_{i}\right)\right)[\mathbf{M}(\sigma \tau) \in D]$.

Such $\sigma$ can be obtained by just choosing a locking sequence $\sigma$ for $\mathbf{M}$ on $L_{i}$, where each of the final grammars have alternated with each other. $D$ can be taken to be the set of final grammars. Here note that $D$ contains a grammar for $L_{i}$.

Let us denote by $\operatorname{Prop}_{i}(\sigma, D)$, the combination of three properties (I), (II), (III).

Now if there exists a $\sigma, D$ satisfying $\operatorname{Prop}_{i}(\sigma, D)$ and $\operatorname{card}\left(W_{i}\right) \geq \max (\{x \mid$ $\langle i, x\rangle \in \operatorname{content}(\sigma)\}$ ), then $W_{i}$ is infinite (otherwise on $\sigma$, which is a member 
of $\operatorname{SEG}\left(S_{i}\right), \mathbf{M}$ returns to a conjecture for $L_{i}$ with grammar for $S_{i}$ in between; to see this note that $D$ contains a grammar for both $S_{i}$ and $L_{i}$ and by clause (II) above, $\mathbf{M}$ alternates between grammars for $L_{i}$ and $S_{i}$ on prefixes of $\sigma$ ).

On the other hand, if there do not exist $\sigma$ and $D$ satisfying $\operatorname{Prop}_{i}(\sigma, D)$ and $\operatorname{card}\left(W_{i}\right) \geq \max (\{x \mid\langle i, x\rangle \in \operatorname{content}(\sigma)\})$, then clearly, $\operatorname{card}\left(W_{i}\right)$ is finite (since $\sigma, D$ satisfying $\operatorname{Prop}_{i}(\sigma, D)$ would then show the finiteness of $W_{i}$ ).

The check whether

$$
(\exists \sigma, D)\left[\operatorname{Prop}_{i}(\sigma) \text { and } \operatorname{card}\left(W_{i}\right) \geq \max (\{x \mid\langle i, x\rangle \in \operatorname{content}(\sigma)\})\right]
$$

is a $\Sigma_{2}$ property. This gives us the desired contradiction.

Corollary 32 For all $a \geq 2, \operatorname{OGDFex}_{a} \subset \operatorname{TxtFex}_{a}$.

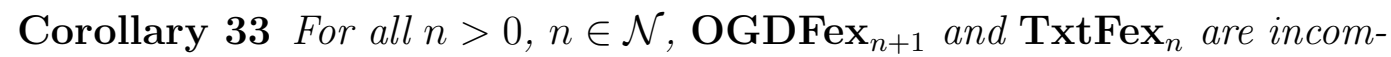
parable.

\section{Behaviourally Correct Learning}

Our first result shows that, in the context of TxtBc-learnability, similarly to TxtEx-learnability, disallowing returning to wrong conjectures significantly limits the power of a learner: even TxtEx-learners can sometimes learn more than any TxtBc-learner that is not allowed returning to wrong conjectures. The reason is that the class $\mathcal{L}$ in $\mathbf{T x t E x}-$ DecBc from [3] contains two distinct extensions of every finite set and thus the next theorem follows from Lemma 20.

\section{Theorem 34 TxtEx $\nsubseteq$ WrDBc.}

Now we compare non U-shaped learning (when a learner cannot abandon a correct conjecture) with learning by disallowing returning to wrong conjectures. From the previous Theorem and from the fact that TxtEx $=$ NUShEx $\subseteq$ NUShBc, we have the following.

Corollary 35 NUShBc $\nsubseteq$ WrDBc.

We now show that, interestingly, the converse is true: WrD learners can sometimes do better than NUSh learners in the TxtBc setting. So WrD and NUSh are incomparable restrictions in the context of TxtBc-identification.

\section{Theorem 36 WrDBc $\nsubseteq$ NUShBc.}

Proof. The proof uses the same class as in the proof of $\mathbf{T x t B c} \neq \mathbf{D e c B c}$ from [17]. The proof that this class witnesses the theorem is a minor modification of the proof of Fulk, Jain, and Osherson [17]. We give the details for completeness.

Let $\mathbf{M}_{0}, \mathbf{M}_{1}, \ldots$ denote a recursive enumeration of total learning machines, where for all $\mathcal{L} \in \mathbf{N U S h B c}$, there exists a $j$ such that $\mathcal{L} \subseteq \operatorname{NUShBc}\left(\mathbf{M}_{j}\right)$. One can construct such an enumeration of total learning machines as done for the TxtEx case (for example, see [25]). 
Let $L_{j}=\{\langle j, x\rangle \mid x \in \mathcal{N}\}$. Let $\sigma_{j, k}=(\langle j, 0\rangle, \ldots,\langle j, k\rangle), L_{j, k}^{1}=\{\langle j, i\rangle \mid i \leq k\}$, and $L_{j, k}^{2}=W_{\mathbf{M}_{j}\left(\sigma_{j, k}\right)}$.

Let $P(j, k)$ be the property that $L_{j, k}^{1} \subset L_{j, k}^{2} \subseteq L_{j}$.

If $(\exists k)[P(j, k)]$, then let $k_{j}$ be the least $k$ such that $P(j, k)$ holds, and then let $\mathcal{S}_{j}=\left\{L_{j, k_{j}}^{1}, L_{j, k_{j}}^{2}\right\} ;$ otherwise, let $\mathcal{S}_{j}=\left\{L_{j}\right\}$. Let $\mathcal{L}=\bigcup_{j \in \mathcal{N}} \mathcal{S}_{j}$.

We will show that $\mathcal{L} \in \mathbf{W r D B c}-\mathbf{N U S h B c}$.

The proof of $\mathcal{L} \in \mathbf{W r D B c}$ is based on utilization of the fact that, if $(\exists k)\left[L_{j, k}^{1} \subset\right.$ $\left.L_{j, k}^{2} \subseteq L_{j}\right]$, then the least such $k$ can be found in the limit.

Claim $37 \mathcal{L} \in$ WrDBc.

Proof. Note that $L \in \mathcal{S}_{j} \Rightarrow L \subseteq L_{j}$.

Let $\operatorname{Cand}_{j}^{n}=\left\{k \leq n \mid L_{j, k}^{1} \subset W_{\mathbf{M}_{j}\left(\sigma_{j, k}\right), n} \subseteq L_{j}\right\}$.

Consider $\mathbf{M}$ which behaves as follows:

$\mathbf{M}$ on input $T[n]$

If content $(T[n])=\emptyset$

Then output a grammar for $\emptyset$.

Else let $j$ be such that content $(T[n]) \subseteq L_{j}$.

(* If there is no such $j$, then the input language is not in the class $\mathcal{L}$. $^{*}$ )

If $\operatorname{Cand}_{j}^{n}=\emptyset$

Then let $\mathbf{M}(T[n])$ be a grammar for $L_{j}$.

Else

Let $k_{j}^{n}=\min \left(\operatorname{Cand}_{j}^{n}\right)$;

If content $(T[n]) \subseteq L_{j, k_{j}^{n}}^{1}$

Then let $\mathbf{M}(T[n])=f\left(j, k_{j}^{n}, n\right)$, where $f$ is as defined below.

Else let $\mathbf{M}(T[n])=g\left(j, k_{j}^{n}, n\right)$, where $g$ is as defined below.

Endif

\section{Endif}

\section{Endif}

\section{End $\mathbf{M}$}

In the above, $f$ and $g$ are such that:

$$
\begin{gathered}
W_{f(j, k, n)}= \begin{cases}L_{j, k}^{1}, & \text { if }(\forall m>n)\left[\min \left(\operatorname{Cand}_{j}^{m}\right)=\min \left(\operatorname{Cand}_{j}^{n}\right)\right] ; \\
L_{j}, & \text { otherwise. }\end{cases} \\
W_{g(j, k, n)}= \begin{cases}L_{j, k}^{2} \cap L_{j}, & \text { if }(\forall m>n)\left[\min \left(\operatorname{Cand}_{j}^{m}\right)=\min \left(\text { Cand }_{j}^{n}\right)\right] ; \\
L_{j}, & \text { otherwise. }\end{cases}
\end{gathered}
$$

We claim that $\mathbf{M}$ WrDBc-identifies $\mathcal{L}$. Let $T$ be a text for $L \in \mathcal{S}_{j}$. Now consider the following cases.

Case 1: $(\forall k)[\neg P(j, k)]$. 
In this case $L=L_{j}$. Furthermore, for all $n$, either $\operatorname{Cand}_{j}^{n}=\emptyset$ or there exists an $m>n$ such that $\min \left(\operatorname{Cand}_{j}^{n}\right) \neq \min \left(\operatorname{Cand}_{j}^{m}\right)$. Thus, if $\mathbf{M}$ outputs $f(j, k, n)$ or $g(j, k, n)$, then $\left[W_{f(j, k, n)}=W_{g(j, k, n)}=L_{j}\right]$. Thus $\mathbf{M}$ on $T[n]$ always outputs a grammar for $L_{j}$, except for the case when $\operatorname{content}(T[n])=\emptyset$. Thus, $\mathbf{M}$ WrDBc-identifies $L$.

Case 2: $P(j, k)$ holds for some $k$.

Let $k_{j}$ be minimal such that $P(j, k)$ holds. Let $m$ be minimal such that for all $n>m, \min \left(\operatorname{Cand}_{j}^{n}\right)=\min \left(\operatorname{Cand}_{j}^{m}\right)=k_{j}$.

Now, for $n<m$, either $\operatorname{Cand}_{j}^{n}=\emptyset$ or for some $n^{\prime}>n, \min \left(\operatorname{Cand}_{j}^{n}\right) \neq$ $\min \left(\operatorname{Cand}_{j}^{n^{\prime}}\right)$. Thus, if content $(T[n]) \neq \emptyset$, then by definition of $\mathbf{M}$ and $f(j, k, n)$ and $g(j, k, n)$, the grammar output by $\mathbf{M}(T[n])$ is for $L_{j}$.

For $n \geq m$, such that $\operatorname{content}(T[n]) \neq \emptyset, \mathbf{M}(T[n])$, outputs $f\left(j, k_{j}, n\right)$ or $g\left(j, k_{j}, n\right)$, based on whether content $(T[n]) \subseteq L_{j, k_{j}}^{1}$ or not.

Thus, if $L=L_{j, k_{j}}^{1}$, then the sequence of grammars output by $\mathbf{M}$ on $T$ are initially for $\emptyset$ (while content $(T[n])=\emptyset$ ), followed by grammars for $L_{j}$ (while $n<m$ and content $(T[n]) \neq \emptyset$ ), and eventually for $L_{j, k_{j}}^{1}$ (when $n \geq m$ and content $(T[n]) \neq \emptyset)$. Thus, $\mathbf{M} \mathbf{W r D B c}$-identifies $L_{j, k_{j}}^{1}$.

On the other hand, if $L=L_{j, k_{j}}^{2}$, then the sequence of grammars output by $\mathbf{M}$ on $T$ are initially for $\emptyset$ (while content $(T[n])=\emptyset$ ), followed by grammars for $L_{j}$ (while $n<m$ and content $(T[n]) \neq \emptyset$ ), followed by grammars for $L_{j, k_{j}}^{1}$ (while $n \geq m$ and $\emptyset \subset$ content $\left.(T[n]) \subseteq L_{j, k_{j}}^{1}\right)$, and then eventually grammars for $L_{j, k_{j}}^{2}$ (when $n \geq m$ and content $(T[n]) \not L_{j, k_{j}}^{1}$ ). Thus, again $\mathbf{M} \mathbf{W r D B c}$-identifies $L$. Note that $L_{j, k_{j}}^{2}$ might be equal to $L_{j}$, and thus decisiveness does not hold. Claim $38 \mathcal{L} \notin$ NUShBc.

Proof. Suppose by way of contradiction that machine $\mathbf{M}_{j} \mathbf{N U S h B c - i d e n t i f i e s}$ $\mathcal{L}$.

Now consider $\mathcal{S}_{j}$.

If $(\forall k)[\neg P(j, k)]$, then $L_{j} \in \mathcal{L}$ which is not $\mathbf{T x t B c}$-identified by $\mathbf{M}_{j}$.

If $(\exists k)[P(j, k)]$, then let $k_{j}$ be the least such $k$. Now $L_{j, k_{j}}^{1}, L_{j, k_{j}}^{2} \in \mathcal{L}$. Since on $\sigma_{j, k_{j}} \mathbf{M}_{j}$ outputs a grammar for $L_{j, k_{j}}^{2} \neq L_{j, k_{j}}^{1}$, there must be extension $\sigma$ of $\sigma_{j, k_{j}}$ such that content $(\sigma)=L_{j, k_{j}}^{1}$ and $W_{\mathbf{M}_{j}(\sigma)}=L_{j, k_{j}}^{1}$. Also there must be an extension $\sigma^{\prime}$ of $\sigma$, such that $\operatorname{content}\left(\sigma^{\prime}\right) \subseteq L_{j, k_{j}}^{2}$ and $W_{\mathbf{M}_{j}\left(\sigma^{\prime}\right)}=L_{j, k_{j}}^{2}$ (since $\mathbf{M}_{j}$ identifies both $\left.L_{j, k_{j}}^{1}, L_{j, k_{j}}^{2}\right)$. But then $\mathbf{M}_{j}$ is $U$-shaped on $L_{j, k_{j}}^{2}$. This proves the claim.

The theorem follows from above claims.

Observe that, in contrast to the case of TxtEx and TxtFex-learning, Theorem 36 implies that $\mathbf{W r D B c}$ does not coincide with DecBc. We have in fact the following corollary of Theorem 36 .

Corollary 39 DecBc $\subset$ WrDBc. 
We next show that, as was the case for $\mathbf{E x}$, inverted-U-shapes are redundant for full Bc-learning power. In fact we have NInvUBc $=$ TxtBc. For this, we first establish Corollary 43 below based on work of [21].

Definition 40 [16] $\mathbf{M}$ is said to be rearrangement independent iff for all $\sigma, \tau$ such that $\operatorname{content}(\sigma)=\operatorname{content}(\tau)$ and $|\sigma|=|\tau|, \mathbf{M}(\sigma)=\mathbf{M}(\tau)$.

Definition 41 [21] A sequence $\sigma$ is normalized if $x \in \operatorname{content}(\sigma) \Rightarrow x \leq|\sigma|$.

A text $T$ is normalized if $T[n]$ is normalized for all $n$.

Theorem 42 [21] Suppose $\mathbf{M}$ is given. Then we can effectively define $\mathbf{M}^{\prime}$ such that:

(a) If $L \in \operatorname{TxtBc}(\mathbf{M})$, then for all normalized texts $T$ for $L$, for all but finitely many $n, \mathbf{M}^{\prime}(T[n])$ is a grammar for $L$.

(b) $\mathbf{M}^{\prime}$ is rearrangement independent.

Corollary 43 Suppose $\mathcal{L} \in \mathbf{T x t B c}$. Then there exists a machine $\mathbf{M}^{\prime}$ such that $\mathbf{M}^{\prime}$ TxtBc-identifies $\mathcal{L}$, and every text $T$ for $L \in \mathcal{L}$ starts with a $\mathbf{T x t B c}$ locking sequence for $\mathbf{M}^{\prime}$ on $L$.

Proof. Suppose $\mathbf{M}$ TxtBc-identifies $\mathcal{L}$ on normalized texts and $\mathbf{M}$ is rearrangement independent (by Theorem 42 such $\mathbf{M}$ exists). Let $\mathbf{M}^{\prime}$ be defined as follows. $\mathbf{M}^{\prime}(\sigma)=\mathbf{M}(\tau)$, where $|\tau|=2 *|\sigma|+\max (\operatorname{content}(\sigma))$ and $\operatorname{content}(\tau)=\operatorname{content}(\sigma)$, and $\tau$ is normalized. Clearly, $\mathbf{M}^{\prime}$ is rearrangement independent.

Now consider any text $T$ for $L \in \mathcal{L}$. Furthermore, let $\alpha$ be a TxtBc-locking sequence (on normalized texts) for $\mathbf{M}$ on $L$. Let $n$ be such that content $(\alpha) \subseteq$ $\operatorname{content}(T[n])$, and $|\alpha| \leq n$. Consider any $\sigma$ such that content $(\sigma) \subseteq L$. Thus, we have that

$$
\mathbf{M}^{\prime}(T[n] \sigma)=\mathbf{M}\left(\alpha \#^{r} T[n] \sigma\right),
$$

where $r=|\sigma|+n-|\alpha|+\max (\operatorname{content}(T[n] \sigma))$. Thus, $\mathbf{M}^{\prime}(T[n] \sigma)$ is a grammar for $L$. Hence, $T[n]$ is a $\mathbf{T x t B c}-$ locking sequence for $\mathbf{M}^{\prime}$ on $L$ and $\mathbf{M}^{\prime} \mathbf{T x t B c}$ identifies $L$ on $T$.

Theorem 44 TxtBc $\subseteq$ NInvUBc.

Proof. Suppose M TxtBc-identifies $\mathcal{L}$. Without loss of generality (by Corollary 43) assume that every text for $L \in \mathcal{L}$ starts with a TxtBc-locking sequence for $\mathbf{M}$ on $L$. By the s-m-n Theorem [28], there exists a recursive function $f$ such that $W_{f(\sigma)}=\bigcup_{s \in \mathcal{N}} A_{\sigma}^{s}$, where $A_{\sigma}^{s}$ is defined as follows.

$A_{\sigma}^{0}=\operatorname{content}(\sigma)$.

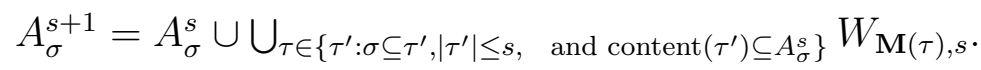

Intuitively, $W_{f(\sigma)}$ is the smallest set $S$ such that $S$ contains content $(\sigma)$ and $W_{\mathbf{M}(\tau)}$ for every $\tau$ satisfying: $\sigma \preceq \tau$, and $\operatorname{content}(\tau) \subseteq S$.

Let $\mathbf{M}^{\prime}(\sigma)=f(\sigma)$.

Now, it is easy to verify that if $\sigma$ is a TxtBc-locking sequence for $\mathbf{M}$ on $L$, then $W_{f(\sigma \tau)}=L$, for any $\tau$ such that $\operatorname{content}(\tau) \subseteq L$. Thus, using the property 
that every text $T$ for $L$ starts with a $\mathbf{T x t B c}$-locking sequence for $\mathbf{M}$ on $L$, we have that $\mathbf{M}^{\prime}$ TxtBc-identifies $\mathcal{L}$.

The following claim follows from the closure property of $W_{f(\sigma)}$.

Claim 45 If $\sigma \preceq \tau$, and $\operatorname{content}(\tau) \subseteq W_{f(\sigma)}$, then $W_{f(\tau)} \subseteq W_{f(\sigma)}$.

Now suppose $T$ is a text for $L \in \mathcal{L}$, and $\sigma \preceq \tau \preceq \gamma \preceq T$, are such that $W_{f(\sigma)}=W_{f(\gamma)} \neq W_{f(\tau)}$. Then, we have

(I) $\operatorname{content}(\tau) \subseteq \operatorname{content}(\gamma) \subseteq W_{f(\gamma)}=W_{f(\sigma)}$ and thus, by Claim $45 W_{f(\tau)} \subseteq$ $W_{f(\sigma)}$.

(II) If content $(\gamma) \subseteq W_{f(\tau)}$ then by Claim $45, W_{f(\gamma)} \subseteq W_{f(\tau)}$, and thus using (I) we would have $W_{f(\tau)}=W_{f(\sigma)}$. A contradiction. Thus, content $(\gamma) \nsubseteq W_{f(\tau)}$. It immediately follows from (II) that $W_{f(\tau)}$ is not a grammar for $L$.

It follows from above analysis that $\mathbf{M}^{\prime}$ NInvUBc-identifies $\mathcal{L}$.

Our next goal is to show that any TxtBc-learner can be transformed into one that does not return to overinclusive conjectures. For this, we first establish Lemmas 46 and 47.

Lemma 46 Suppose $\mathbf{M}$ is given. Then, for any $\sigma \in S E Q$, there exists an r.e. set $P(\sigma)$ such that

- A grammar for $P(\sigma)$ can be effectively obtained from $\sigma$;

- If $\sigma$ is a $\mathbf{T x t B c - l o c k i n g ~ s e q u e n c e ~ f o r ~} \mathbf{M}$ on $W_{\mathbf{M}(\sigma)}$, then $P(\sigma)$ contains only grammars for $W_{\mathbf{M}(\sigma)}$;

- If $\sigma$ is not a $\mathbf{T x t B c}$-locking sequence for $\mathbf{M}$ on $W_{\mathbf{M}(\sigma)}$, then $P(\sigma)$ is either empty, or contains grammars for at least two distinct languages.

Proof. Define $P(\sigma)$ as follows.

If content $(\sigma) \nsubseteq W_{\mathbf{M}(\sigma)}$, then let $P(\sigma)=\emptyset$.

Otherwise let $P(\sigma)=\left\{\mathbf{M}(\tau) \mid \sigma \preceq \tau\right.$, content $\left.(\tau) \subseteq W_{\mathbf{M}(\sigma)}\right\}$.

Now if $\sigma$ is a TxtBc-locking sequence for $\mathbf{M}$ on $W_{\mathbf{M}(\sigma)}$, then $P(\sigma)$ consists only of grammars for $W_{\mathbf{M}(\sigma)}$. On the other hand if $\sigma$ is not a TxtBc-locking sequence for $\mathbf{M}$ on $W_{\mathbf{M}(\sigma)}$, then either $P(\sigma)$ is empty or it contains grammars for at least two distinct languages.

Lemma 47 Suppose $\mathbf{M}$ is given. Then, there exists a recursive function $g$ such that:

(a) If $\sigma$ is a $\mathbf{T x t B c - l o c k i n g ~ s e q u e n c e ~ f o r ~} \mathbf{M}$ on $W_{\mathbf{M}(\sigma)}$, then $W_{g(\sigma)}=W_{\mathbf{M}(\sigma)}$.

(b) If $\sigma$ is not a $\mathbf{T x t B c - l o c k i n g ~ s e q u e n c e ~ f o r ~} \mathbf{M}$ on $W_{\mathbf{M}(\sigma)}$, then $W_{g(\sigma)}$ is finite.

Proof. For a finite set $X$ and number $s$, let

- $\operatorname{CommonTime}(X, s)=\max \left(\left\{t \leq s \mid\left(\forall p, p^{\prime} \in X\right)\left[W_{p, t} \subseteq W_{p^{\prime}, s}\right]\right\}\right)$;

- $\operatorname{CommonElem}(X, s)=\bigcap_{p \in X} W_{p, \operatorname{CommonTime}(X, s)}$.

Let $f$ be a recursive function such that, $W_{f(X)}=\bigcup_{s \in \mathcal{N}} \operatorname{CommonElem}(X, s)$. Here we assume that $W_{f(\emptyset)}=\emptyset$. 
Intuitively, CommonTime $(X, s)$ finds the largest time $t$ such that all elements enumerated up to time $t$ by some grammars in $X$ are included in all languages enumerated by grammars in $X$ up to time s. CommonElem $(X, s)$ then gives the set of the elements enumerated by all grammars in $X$ up to time CommonTime $(X, s)$. Note that

(I) $\lim _{s \rightarrow \infty}$ CommonTime $(X, s)$ is infinite iff all grammars in $X$ are for the same language;

(II) If $X \subseteq X^{\prime}$, then CommonTime $(X, s) \geq \operatorname{CommonTime}\left(X^{\prime}, s\right)$;

(III) If $W_{p} \neq W_{p^{\prime}}$ then for all $s$, CommonTime $\left(\left\{p, p^{\prime}\right\}, s\right)$ is bounded by the least $t$ such that $W_{p, t} \cup W_{p^{\prime}, t} \nsubseteq W_{p} \cap W_{p^{\prime}}$.

Suppose $X_{0} \subseteq X_{1} \subseteq \ldots$ is given. Let $Y$ be the set of all $y$ such that there is an $s \geq y$, such that $y \in W_{f\left(X_{s}\right)}$. Note that (II) and (III) above imply that if $\left\{p, p^{\prime}\right\} \subseteq \cup_{i \in \mathcal{N}} X_{i}$ and $W_{p} \neq W_{p^{\prime}}$, then $Y$ is finite. On the other hand, if all $p, p^{\prime} \in \bigcup_{i \in \mathcal{N}} X_{i}$ are grammars for the same language, then $Y=W_{p}$ for any $p \in \bigcup_{i \in \mathcal{N}} X_{i}$.

Let $P$ be as in Lemma 46 and let $P_{s}(\sigma)$ denote $P(\sigma)$ enumerated in $s$ steps.

Now let $g(\sigma)$ be such that $W_{g(\sigma)}=\bigcup_{s \in \mathcal{N}}\left[\left\{y \mid y \leq s \wedge y \in W_{f\left(P_{s}(\sigma)\right)}\right\}\right]$. It is now easy to verify that Lemma holds.

Now we can prove one of our main results: any TxtBc-learner can be replaced by one not returning to overinclusive conjectures.

\section{Theorem 48 TxtBc $\subseteq$ OIDBc.}

Proof. Suppose M TxtBc-identifies $\mathcal{L}$. Without loss of generality (Corollary 43) assume that for any text $T$ for $L \in \mathcal{L}$, there exists a $\sigma \preceq T$, such that $\sigma$ is a $\mathbf{T x t B c}$-locking sequence for $\mathbf{M}$ on $L$. Intuitively, the proof employs two tricks. The first trick (as given by $g$ in Lemma 47) is to make sure that the learner outputs a conjecture for an infinite language only on $\sigma$ 's which are TxtBc-locking sequences for the conjectured language. This automatically ensures that no semantic mind changes occur between different grammars output for the same infinite language by the learner. The second trick makes sure that all finite languages that are conjectured by the learner and that go beyond what is seen in the input at the time of conjecture, are pairwise distinct.

We now proceed formally.

Let $g$ be as in Lemma 47 .

Let $q_{0}, q_{1}, \ldots$ denote an increasing sequence of primes.

Now define $\mathbf{M}^{\prime \prime}$ as follows. $\mathbf{M}^{\prime \prime}(\sigma)=h(\sigma)$, where $W_{h(\sigma)}$ is defined as follows. We assume without loss of generality that for all $i$ and $s, W_{i, s+1}-W_{i, s}$ contains at most one element. This ensures that when $W_{g(\sigma)}$ is infinite, then $\operatorname{card}\left(W_{g(\sigma), s}\right)$ would be of form $\left(q_{\text {ind }(\sigma)}\right)^{k}$, for infinitely many $s$.

$W_{h(\sigma)}$

1. Enumerate content $(\sigma)$ 
2. Loop

Search for $s$ such that $W_{h(\sigma)}$ enumerated up to now is a proper subset of $W_{g(\sigma), s}$, and $\operatorname{card}\left(W_{g(\sigma), s}\right)$ is $\left(q_{\operatorname{ind}(\sigma)}\right)^{k}$ for some $k$.

If and when such $s$ is found, enumerate $W_{g(\sigma), s}$.

Forever

End

Thus, $W_{h(\sigma)}$ is $W_{g(\sigma)}$ if $W_{g(\sigma)}$ is infinite. Furthermore, if $W_{h(\sigma)}$ is finite, then it is either content $(\sigma)$ or has cardinality a power of $q_{\operatorname{ind}(\sigma)}$.

It follows that if $W_{h(\sigma)}=W_{h(\tau)}$, for $\sigma \prec \tau$, then either $W_{h(\sigma)}$ is infinite and $\sigma$ is a TxtBc-locking sequence for $\mathbf{M}$ on $W_{g(\tau)}=W_{g(\sigma)}=W_{h(\sigma)}$, and thus, there is no semantic mind change by $\mathbf{M}^{\prime \prime}$ in between $\sigma$ and $\tau$, or $W_{h(\sigma)}$ is finite, and thus, it must be the case that $W_{h(\sigma)}=W_{h(\tau)}=\operatorname{content}(\tau)$ (otherwise, $q_{\text {ind }(\sigma)} \neq q_{\text {ind }(\tau)}$ would imply that $\left.W_{h(\sigma)} \neq W_{h(\tau)}\right)$.

It follows from above cases that $\mathbf{M}^{\prime \prime}$ does not return to overinclusive hypotheses. To see TxtBc-identification of $L \in \mathcal{L}$, let $T$ be a text for $L$. Let $T[n]$ be a TxtBc-locking sequence for $\mathbf{M}$ on $L$ (such an $n$ exists by assumption on $\mathbf{M}$ ). Thus, $g(T[n])$ is a grammar for $L$. If $L$ is finite, then without loss of generality we also assume that $n$ is large enough such that $L \subseteq \operatorname{content}(T[n])$. Now consider any $m \geq n$. It is easy to verify that if $L$ is infinite then $W_{h(T[m])}=W_{g(T[m])}=L$. On the other hand, if $L$ is finite, then again $W_{h(T[m])}$ does not enumerate anything beyond first step, and thus equals $L$.

\section{Corollary 49 TxtBc $\subseteq$ OGDBc.}

\section{Consistency}

Consistency is a natural and important requirement in the context of TxtExand of TxtBc-learning. While, for the latter, consistency can be easily achieved, it is known to be restrictive for TxtEx-learnability [4,25]. In this section we establish a new interesting boundary on consistent TxtEx-learnability - in Theorem 53 we show that consistent TxtEx-learners can be made consistent and decisive - contrast this result with Theorem 24 .

Definition $50[4,25] \mathbf{M}$ is said to be consistent on $T$ iff, for all $n, \mathbf{M}(T[n]) \downarrow$ and content $(T[n]) \subseteq W_{\mathbf{M}(T[n])}$.

$\mathbf{M}$ is said to be consistent on $L$ iff, $\mathbf{M}$ is consistent on each text for $L$.

Definition 51 (a) [4,25] $\mathbf{M}$ ConsTxtEx-identifies $L$ iff $\mathbf{M}$ is consistent on $L$, and $\mathbf{M}$ TxtEx-identifies $L$.

(b.1) [4] M ConsTxtEx-identifies $\mathcal{L}$ iff $\mathbf{M}$ ConsTxtEx-identifies each $L \in \mathcal{L}$.

(b.2) ConsTxtEx $=\{\mathcal{L} \mid(\exists \mathbf{M})[\mathbf{M}$ ConsTxtEx-identifies $\mathcal{L}]\}$. 
Note that for $\mathbf{M}$ to ConsTxtEx-identify a text $T$, it must be defined on each initial segment of $T$. One can similarly define a combination of consistency with decisiveness (called ConsDecEx) and other related criteria such as ConsNUShEx, ConsOIDEx, ConsWrDEx, etc.

Two other versions of consistency have been considered in the literature, namely $\mathcal{R}$ Cons [20], where the learner must be total but might be inconsistent on data not belonging to the class to be learned, and $\mathcal{T}$ Cons [32], where the learner must be total and consistent on every text, whether it is for some language to be learned or not.

Our simulations results below (Theorem 53 to Corollary 58) hold for $\mathcal{T}$ Cons replacing Cons. We do not yet know whether Theorem 53 holds for $\mathcal{R}$ Cons, and correspondingly whether Corollary 58 also holds for $\mathcal{R}$ Cons. Theorem 57 does hold for $\mathcal{R}$ Cons also. Theorems 54, 55, and 56 hold for $\mathcal{T}$ Cons, and thus for $\mathcal{R}$ Cons too.

Our diagonalization results Theorem 60 and Theorem 61(a) also hold for $\mathcal{T}$ Cons. Theorem $61(\mathrm{~b})$ holds for $\mathcal{R}$ Cons, but is known not to hold for $\mathcal{T}$ Cons replacing Cons. We omit the details, and will not consider $\mathcal{R}$ Cons and $\mathcal{T}$ Cons from now on.

Definition 52 We say that $\sigma$ is self-stabilizing for $\mathbf{M}$ if $\sigma$ is a TxtEx-locking sequence for $\mathbf{M}$ on $W_{\mathbf{M}(\sigma)}$.

Theorem 53 ConsTxtEx $\subseteq$ ConsDecEx.

Proof. Suppose M ConsTxtEx-identifies $\mathcal{L}$. An easy modification of the proof of Lemma 19 of the current paper (along with corresponding modification of Lemma 17) can be used to show that, if there exists a finite set $A$ such that no extension of $A$ is in $\mathcal{L}$ then $\mathcal{L} \in$ ConsDecEx.

On the other hand, if $\mathcal{L}$ contains an extension of every finite set $A$, then $\mathbf{M}$ is total, and consistent on all inputs. Now, for each $\sigma$, we define:

$$
F_{\sigma}(x)= \begin{cases}1, & \text { if } \mathbf{M}(\sigma x)=\mathbf{M}(\sigma) \\ 0, & \text { otherwise }\end{cases}
$$

Clearly, $F_{\sigma}$ is total for each $\sigma$. Furthermore, if $\sigma$ is self-stabilizing for $\mathbf{M}$, then $F_{\sigma}^{-1}(1)=W_{\mathbf{M}(\sigma)}$. Thus, $\mathcal{L} \subseteq\left\{F_{\sigma}^{-1}(1) \mid \sigma \in \mathrm{SEQ}\right\}$.

Let $G(2 x)=0$ and $G(2\langle i, x\rangle+1)=1-F_{\delta_{i}}(2\langle i, x\rangle+1)$. Thus, for all $\sigma, G$ is not a finite variant of $F_{\sigma}$ (that is, $G$ differs from $F_{\sigma}$ on infinitely many inputs), and $G$ is 0 on all even inputs.

Let $s_{\sigma}=\min \left(\left\{t \mid(\exists \tau)\left[\operatorname{content}(\tau) \subseteq W_{\mathbf{M}(\sigma), t} \wedge|\tau| \leq t \wedge \mathbf{M}(\sigma) \neq \mathbf{M}(\sigma \tau)\right]\right\}\right)$. Thus, $s_{\sigma}=\infty$ iff $\sigma$ is self-stabilizing for $\mathbf{M}$. Moreover, one can effectively determine if $s_{\sigma} \leq t$, for any given $t$. Now define $h(\sigma), g(\sigma)$ as follows. 


$$
\begin{aligned}
& \varphi_{h(\sigma)}(x)= \begin{cases}F_{\sigma}(x), & \text { if } x \leq s_{\sigma} ; \\
G(x), & \text { if } x>s_{\sigma} \text { and } x \text { is odd; } \\
1, & \text { if } x>s_{\sigma} \text { and } x=2 *\left\langle 2 * \operatorname{ind}(\sigma), 1+s_{\sigma}\right\rangle ; \\
0, & \text { otherwise; }\end{cases} \\
& \varphi_{g(\sigma)}(x)= \begin{cases}1, & \text { if } x \in \operatorname{content}(\sigma) ; \\
G(x), & \text { if } x \notin \operatorname{content}(\sigma) \text { and } x \text { is odd; } \\
1, & \text { if } x=2 *\langle 2 * \operatorname{ind}(\sigma)+1, \max (\operatorname{content}(\sigma))\rangle ; \\
0, & \text { otherwise. }\end{cases}
\end{aligned}
$$

Intuitively, the aim of $\varphi_{h(\sigma)}$ is to follow $F_{\sigma}$, if $\sigma$ is self-stabilizing. Otherwise, it computes a finite variant of $G$. The third clause in the definition of $\varphi_{h(\sigma)}$ above is used to ensure that the $\varphi_{h(\sigma)}$ 's which compute a finite variant of $G$ are pairwise distinct. $g(\sigma)$ is used below only for maintaining consistency, in case one cannot find an appropriate program $h(\sigma)$. Again, the third clause in the definition of $\varphi_{g(\sigma)}$ is to ensure that different $\varphi_{g(\sigma)}$ 's and $\varphi_{h(\sigma)}$ 's which compute a finite variant of $G$ are pairwise distinct.

It can be easily verified, using the definition of $g$ and $h$ above, that

(1) If $\sigma$ is self-stabilizing for $\mathbf{M}$, then $\varphi_{h(\sigma)}=F_{\sigma}$.

(2) If $\sigma$ is not self-stabilizing for $\mathbf{M}$, then $\varphi_{h(\sigma)}$ is a finite variant of $G$ (that is, $\varphi_{h(\sigma)}$ and $G$ differ only on finitely many inputs $)$, and $\max \left(\left\{x \mid \varphi_{h(\sigma)}(2 x)=1\right\}\right)$ is of form $\langle 2 * \operatorname{ind}(\sigma), \cdot\rangle$. Note that $2 * \operatorname{ind}(\sigma)$ in the pair makes function $\varphi_{h(\sigma)}$ different from $\varphi_{g(\sigma)}$ and $\varphi_{h\left(\sigma^{\prime}\right)} / \varphi_{g\left(\sigma^{\prime}\right)}$, for $\sigma \neq \sigma^{\prime}$.

(3) $\varphi_{g(\sigma)}$ is a finite variant of $G$, and $\max \left(\left\{x \mid \varphi_{g(\sigma)}(2 x)=1\right\}\right)$ is of form $\langle 2 * \operatorname{ind}(\sigma)+1, \cdot\rangle$. Note that $2 * \operatorname{ind}(\sigma)+1$ in the pair makes function $\varphi_{g(\sigma)}$ different from $\varphi_{h(\sigma)}$ and $\varphi_{h\left(\sigma^{\prime}\right)} / \varphi_{g\left(\sigma^{\prime}\right)}$, for $\sigma \neq \sigma^{\prime}$.

Now define $\mathbf{M}^{\prime}$ on $T$ as follows. Let

$$
\sigma_{\langle i, j\rangle}= \begin{cases}\delta_{i}, & \text { if } \operatorname{content}\left(\delta_{i}\right) \subseteq \operatorname{content}(T[\langle i, j\rangle]) \\ \lambda, & \text { otherwise }\end{cases}
$$

Above gives a special enumeration of all finite segments whose content are contained in content $(T)$.

Let gram be a recursive function such that $W_{\text {gram }(i)}=\varphi_{i}^{-1}(1)$.

$$
\mathbf{M}^{\prime}(T[n])= \begin{cases}\operatorname{gram}\left(h\left(\sigma_{m_{n}}\right)\right), & \text { for the least } m_{n} \leq n \text { such that } \\ & s_{\sigma_{m_{n}}} \geq n, \text { and } \\ & \operatorname{content}(T[n]) \subseteq F_{\sigma_{m_{n}}}^{-1}(1), \text { and } \\ & \text { content }(T[n]) \subseteq \varphi_{h\left(\sigma_{m_{n}}\right)}^{-1}(1) ; \\ \operatorname{gram}(g(T[n])), & \text { otherwise, if there is no such } m_{n} \leq n .\end{cases}
$$

It is easy to verify that $\mathbf{M}^{\prime}$ is consistent. Moreover, $\mathbf{M}^{\prime} \mathbf{T x t E x}$-identifies $\mathcal{L}$, since for any text $T$ for $L \in \mathcal{L}$, for the least $m$ such that $\sigma_{m}$ is a TxtEx-locking sequence for $\mathbf{M}$ on $L, \mathbf{M}^{\prime}$ stabilizes to $\operatorname{gram}\left(h\left(\sigma_{m}\right)\right)$. We now show that $\mathbf{M}^{\prime}$ is decisive. Note that $m_{n}$ (when defined) is increasing in $n$. Suppose, by way 
of contradiction, $W_{\mathbf{M}^{\prime}\left(T\left[n_{1}\right]\right)}=W_{\mathbf{M}^{\prime}\left(T\left[n_{3}\right]\right)} \neq W_{\mathbf{M}^{\prime}\left(T\left[n_{2}\right]\right)}$, where $n_{1}<n_{2}<n_{3}$. Note that if $\mathbf{M}^{\prime}\left(T\left[n_{1}\right]\right)=\operatorname{gram}\left(g\left(T\left[n_{1}\right]\right)\right)$ or $\mathbf{M}^{\prime}\left(T\left[n_{3}\right]\right)=\operatorname{gram}\left(g\left(T\left[n_{3}\right]\right)\right)$, then $W_{\mathbf{M}^{\prime}\left(T\left[n_{1}\right]\right)} \neq W_{\mathbf{M}^{\prime}\left(T\left[n_{3}\right]\right)}$ (by definition of $g(\cdot)$, and properties (2) and (3) above). Thus, $W_{\mathbf{M}^{\prime}\left(T\left[n_{1}\right]\right)}=W_{\operatorname{gram}\left(h\left(m_{n_{1}}\right)\right)}$ and $W_{\mathbf{M}^{\prime}\left(T\left[n_{3}\right]\right)}=W_{\operatorname{gram}\left(h\left(m_{n_{3}}\right)\right)}$. If $m_{n_{1}}=m_{n_{3}}$, then by monotonicity we will also have $m_{n_{2}}=m_{n_{1}}$, and thus $W_{\mathbf{M}^{\prime}\left(T\left[n_{2}\right]\right)}=W_{\mathbf{M}^{\prime}\left(T\left[n_{1}\right]\right)}$. On the other hand, if $m_{n_{1}} \neq m_{n_{3}}$, then $\sigma_{m_{n_{1}}}$ and $\sigma_{m_{n_{3}}}$ must both be self-stabilizing for $\mathbf{M}$, since, otherwise, $W_{\operatorname{gram}\left(h\left(\sigma_{m_{n_{1}}}\right)\right)} \neq$ $\left.W_{\text {gram }\left(h\left(\sigma_{m_{n}}\right)\right.}\right)$ (by $(1),(2)$ above and the fact that $G$ is not a finite variant of $F_{\sigma}$ for any $\sigma)$. But, then content $\left(T\left[n_{3}\right]\right) \not F_{\sigma_{m_{n_{1}}}}^{-1}(1)=W_{h\left(\sigma_{m_{n_{1}}}\right)}$ (by definition of $h$, and the fact that $\sigma_{m_{n_{1}}}$ is not a stabilizing sequence for $\mathbf{M}$ on content $\left(\sigma_{m_{n_{3}}}\right)$ ), a contradiction to $W_{\text {gram }\left(h\left(\sigma_{m_{n_{1}}}\right)\right)}=W_{\text {gram }\left(h\left(\sigma_{m_{n_{3}}}\right)\right)} \supseteq \operatorname{content}\left(T\left[n_{3}\right]\right)$. It follows that $\mathbf{M}^{\prime}$ must be decisive.

\section{Theorem 54 NUShBc $=$ ConsNUShBc.}

Proof. Suppose M NUShBc-identifies $\mathcal{L}$. Let $E(\sigma)=\{\tau \preceq \sigma \mid \operatorname{content}(\tau)=$ content $(\sigma)\}$ and define $\mathbf{M}^{\prime}$ as follows.

$$
W_{\mathbf{M}^{\prime}(\sigma)}= \begin{cases}W_{\mathbf{M}(\sigma)}, & \text { if }(\forall \tau \in E(\sigma))\left[\operatorname{content}(\sigma) \subseteq W_{\mathbf{M}(\tau)}\right] \\ \operatorname{content}(\sigma), & \text { otherwise. }\end{cases}
$$

Clearly, $\mathbf{M}^{\prime}$ is consistent.

We now show that $\mathbf{M}^{\prime}$ NUShBc-identifies $\mathcal{L}$. To see this, consider any text $T$ for $L \in \mathcal{L}$. Let $n$ be the least number such that $W_{\mathbf{M}(T[n])}=L$. It follows from $\mathbf{M}$ being non U-shaped that, for all $m \geq n, L=W_{\mathbf{M}(T[m])}$. We now consider two cases.

Case 1: $L=\operatorname{content}(T[n])$.

In this case, for all $m \geq n, W_{\mathbf{M}^{\prime}(T[m])}=L$, (based on either clause of the definition of $\mathbf{M}^{\prime}$ ). Thus, $\mathbf{M}^{\prime}$ TxtBc-identifies $T$. Now suppose there exists an $m^{\prime}<n$ such that $W_{\mathbf{M}^{\prime}\left(T\left[m^{\prime}\right]\right)}=L$. Then, since $W_{\mathbf{M}\left(T\left[m^{\prime}\right]\right)} \neq L$, we have that $W_{\mathbf{M}^{\prime}\left(T\left[m^{\prime}\right]\right)}(=L)=\operatorname{content}\left(T\left[m^{\prime}\right]\right)=\operatorname{content}(T[n])$, and $\operatorname{content}\left(T\left[m^{\prime}\right]\right) \nsubseteq$ $W_{\mathbf{M}(T[r])}$, for some $r \leq m^{\prime}, T[r] \in E\left(T\left[m^{\prime}\right]\right)$. It follows by definition of $\mathbf{M}^{\prime}$ that for all $m^{\prime \prime}$ such that $m^{\prime} \leq m^{\prime \prime} \leq n, W_{\mathbf{M}^{\prime}\left(T\left[m^{\prime \prime}\right]\right)}=\operatorname{content}\left(T\left[m^{\prime \prime}\right]\right)=L$. It follows that $\mathbf{M}^{\prime}$ is non-U-shaped on $T$.

Case 2: Not Case 1 (that is content $(T[n]) \subset L)$.

In this case, let $n^{\prime} \geq n$ be minimal such that content $(T[n]) \neq \operatorname{content}\left(T\left[n^{\prime}\right]\right)$. Clearly, for all $m \geq n^{\prime}, \mathbf{M}^{\prime}(T[m])$ is a grammar for $L$. Thus, $\mathbf{M}^{\prime} \mathbf{T x t B c}-$ identifies $T$. Furthermore, for all $m \leq n-1, \mathbf{M}^{\prime}(T[m])$ is not a grammar for $L$ (using either clause of the definition of $\mathbf{M}^{\prime}$ ). Furthermore, for all $m$ such that $n \leq m<n^{\prime}, \mathbf{M}^{\prime}(T[m])$ will be a grammar for $L$ iff for all $T[s] \in$ $E(T[n])$, content $(T[n]) \subseteq W_{\mathbf{M}(T[s])}$ (by definition of $\mathbf{M}^{\prime}$, and using the fact that content $(T[n]) \subseteq L=W_{\mathbf{M}\left(T\left[m^{\prime}\right]\right)}$, for $\left.n \leq m^{\prime}<n^{\prime}\right)$. It follows that $\mathbf{M}^{\prime}$ is non-U-shaped on $T$.

We note that the proof of Theorem 44 also shows the following inclusion. 
Theorem 55 TxtBc $\subseteq$ ConsNInvUBc.

The proof of Theorem 48 also shows the following inclusion.

Theorem 56 TxtBc $\subseteq$ ConsOIDBc.

The proof of Theorem 27 also works for the case when we are considering consistent identification.

Theorem 57 (a) ConsWrDFex ${ }_{*} \subseteq$ ConsTxtEx.

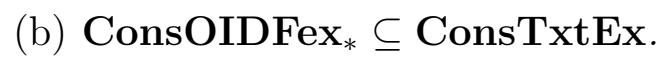

Corollary 58 (a) ConsWrDFex ${ }_{*} \subseteq$ ConsDecEx.

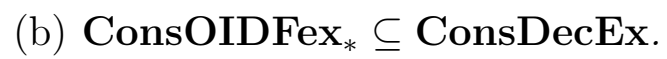

Next we show that decisive learning is stronger than consistent learning.

Theorem 59 DecEx $\nsubseteq$ ConsTxtEx.

Proof. Without loss of generality assume $\varphi_{0}(0) \uparrow\left(\right.$ and thus $\left.\varphi_{0}(0) \neq 0\right)$.

Let $\mathcal{C}=\left\{f \mid\left(\forall^{\infty} x\right)[f(x)=0]\right\} \cup\left\{f \mid f\right.$ is monotonically increasing, $\varphi_{f(0)}=f$, and for all $\left.x, \Phi_{f(0)}(x)<f(x+1)\right\}$. For a function $f$, let $L_{f}=\{\langle x, f(x)\rangle \mid x \in$ $\mathcal{N}\}$. Let $\mathcal{L}=\left\{L_{f} \mid f \in \mathcal{C}\right\}$. It is well known that $\mathcal{C} \notin$ ConsEx (for function version of consistency, see for example [4]), and hence $\mathcal{L} \notin$ ConsTxtEx.

On the other hand, $\mathcal{L} \in$ DecEx can be shown as follows. Let $p$ be a recursive function such that

$$
\varphi_{p(i)}(x)= \begin{cases}i, & \text { if } x=0 \text { and } \varphi_{i}(0)=i ; \\ \varphi_{i}(x), & \text { if } x>0, \varphi_{i}(y) \text { is defined for all } y \leq x, \text { and } \\ \uparrow, & (\forall y<x)\left[\max \left(\left\{\Phi_{i}(y), \varphi_{i}(y)\right\}\right)<\varphi_{i}(y+1)\right] ; \\ \uparrow, & \text { otherwise. }\end{cases}
$$

Now, $\mathbf{M}$ on input $\sigma$ behaves as follows:

$\mathbf{M}(\sigma)$

1. If $\langle 0, e\rangle \notin \operatorname{content}(\sigma)$ for any $e$

Then output a grammar for $\emptyset$.

2. Else If $\left\langle x, y_{0}\right\rangle,\left\langle x, y_{1}\right\rangle \in \operatorname{content}(\sigma)$, for some $x$, and $y_{0} \neq y_{1}$,

Then output a grammar for $\mathcal{N}$.

3. Else

4. Let $\langle 0, e\rangle \in \operatorname{content}(\sigma)$ (here such $e$ is unique).

5. If $\varphi_{e,|\sigma|}(0) \neq e$ or $\left[\operatorname{content}(\sigma)-\operatorname{content}\left(\sigma\left[\Phi_{e}(0)\right]\right) \subseteq\{\langle z, 0\rangle \mid z \in \mathcal{N}\}\right]$,

Then output a grammar for

$$
L=\operatorname{content}(\sigma) \cup\{\langle x, 0\rangle \mid(\forall y>0)[\langle x, y\rangle \notin \operatorname{content}(\sigma)]\} .
$$

6. $\quad$ Else let $m=\max (\{x \mid(\exists y)[\langle x, y\rangle \in \operatorname{content}(\sigma)]\})$.

Let $m^{\prime}$ be such that $\left\langle m, m^{\prime}\right\rangle \in \operatorname{content}(\sigma)$.

7. If $(\forall x \mid 0<x<m)\left[\Phi_{e}(x)<m^{\prime}\right.$ and $\left.\max \left(\left\{\Phi_{e}(x-1), \varphi_{e}(x-1)\right\}\right)<\varphi_{e}(x)\right]$ and $(\forall\langle x, y\rangle \mid x<m,\langle x, y\rangle \in \operatorname{content}(\sigma))\left[\varphi_{e}(x)=y\right]$, 
Then output a grammar for $L_{\varphi_{p(e)}}$.

8. Else Output a grammar for

$$
L=\operatorname{content}(\sigma) \cup\{\langle x, 0\rangle \mid(\forall y>0)[\langle x, y\rangle \notin \operatorname{content}(\sigma)]\}
$$

\section{Endif}

10. Endif

\section{Endif}

\section{End}

It is easy to verify that $\mathbf{M}$ TxtEx-identifies $\mathcal{L}$. To see decisiveness, note that a grammar for $\emptyset$ is output only until some $\langle 0, x\rangle$ appears in the input. Furthermore, if a grammar for $\mathcal{N}$ is output on some $\sigma$, then, for all $\tau \supseteq \sigma$, $\mathbf{M}$ outputs a grammar for $\mathcal{N}$. Once $\langle 0, e\rangle$ appears in the input for some $e$, with $\varphi_{e}(0)=e$ (and it is known that the previous conjecture of $\mathbf{M}$ is wrong, see step 5), $L_{\varphi_{p(e)}}$ is output as long as it is consistent with the input, and it seems that $\varphi_{e} \in\left\{f \mid f\right.$ is monotonically increasing, $\varphi_{f(0)}=f$, and for all $\left.x, \Phi_{f(0)}(x)<f(x+1)\right\}$. Thus, once $L_{\varphi_{p(e)}}$, is abandoned, it is never conjectured again. Furthermore, trivially, outputs in step 5 and 8 are monotonic in the input. Thus, $\mathbf{M}$ is decisive.

We note that the proof of Theorem 36 also shows the following.

\section{Theorem 60 ConsWrDBc $\nsubseteq$ NUShBc.}

The proof of Theorem 31 gives us part (a) of the following theorem. The proof of Theorem 30 can be easily modified to give part (b) of the following theorem (we just need to make the learner given there consistent, assuming that the input language is from the class).

Theorem 61 (a) ConsTxtFex $2 \nsubseteq$ OGDFex $_{*}$.

(b) ConsOGDFex $_{n+1} \nsubseteq$ TxtFex $_{n}$.

\section{Summary and Open Problems}

We summarize our results on the impact of the WrD, NInvU, OID, and OGD variants of non U-shaped behaviour and how they compare to previous results about the original notion NUSh from [3] and [9]. We also tentatively consider their possible significance from a cognitive science perspective.

Returning to abandoned wrong conjectures turned out to be necessary for full learning power in all three of the models TxtEx, TxtFex, and TxtBc. Admittedly, disallowing return to any kind of wrong conjecture is, a priori, quite a strong requirement on a learning machine. Thus, mathematically, these separation results might not be too surprising. However, from the viewpoint of developmental psychology, they seem to suggest a very deep necessity of an 
apparently inefficient learning behaviour. This might also suggest that principles of "economy" are at work in the hypothesis formulation process. The learner might tend to keep a hold on a few different hypotheses, going back and forth between them before converging to a correct one.

It is certainly premature to draw from our results any hypothesis on why returning to wrong conjectures might be necessary for human learning power. We can however note that the necessity of overgeneralization for learning machines, as well as the interplay between learning finite tables and learning possibly infinite sets are, interestingly, a key ingredient in the proof of our separation result for TxtBc, Theorem 36, showing the necessity of returning to wrong conjecture to maintain full learning power. The same is the case for the proof of the necessity of returning to correct conjectures for TxtBc-learners (see $[17,3])$. The key role of overgeneralization - and, in particular, of incorrect overgeneralization - in U-shaped learning phenomena, is also illustrated by our Theorem 53, showing that TxtEx-learners that are consistent with the current data can be made decisive. Analogously, Theorem 54 shows that non U-shaped TxtBc-learners can be made non U-shaped and consistent.

On the other hand, we have shown that inverted-U-shaped learning, returning to abandoned overinclusive conjectures and returning to abandoned overgeneralizing conjectures are necessary only for the vacillatory case and avoidable otherwise. Note that these results, when coupled with the previous separations results, imply that, for example, any TxtEx- or TxtBc-learner that avoids overinclusive conjectures will necessarily return (on some text for some language in some class) to a conjecture for a proper subset of the target language. It might be interesting to further investigate which non U-shaped features can be simultaneously satisfied by a single TxtEx or TxtBc-learner. In particular, whether inverted U-shapes and return to overinclusive conjectures can be simultaneously avoided for all classes in TxtEx or TxtBc. This analysis could give us more information on what the reason for the necessity of returning to wrong conjectures might be.

The above summarized results can be compared to results in [3] and [9] showing that returning to abandoned correct conjectures is avoidable in the TxtEx case while being necessary for vacillatory and behaviourally correct identification. The results of [9] and of the present paper have proved that the vacillatory learning hierarchy is extremely sensitive to non U-shaped restrictions. Instead, explanatory and behaviourally correct learning are sensitive only to the strongest forms of U-shaped learning considered, i.e., to the decisive and the wrong-decisive restrictions. This might be seen as an argument in favour of vacillatory learning as a possible candidate model of human learning. As already observed, the capacity of a candidate learning model to account for U-shaped learning is widely recognized in the cognitive science literature as an argument in favour of the proposed model.

Also, we can conclude that disallowing returning to abandoned wrong conjec- 


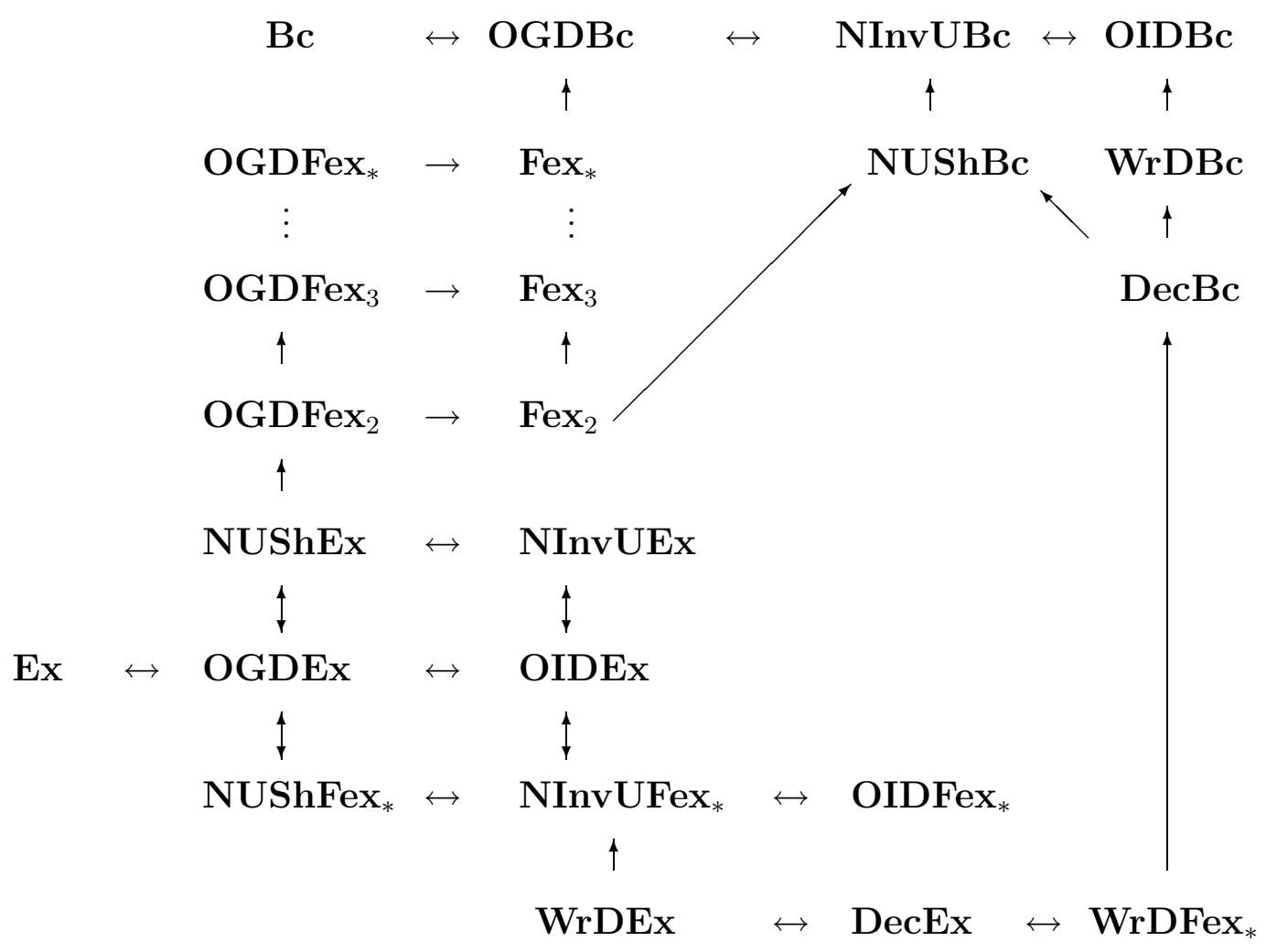

Fig. 1. Summary of the results. We have dropped the word Txt from the criteria TxtEx, $\mathbf{T x t F e x}_{a}$, TxtBc for ease of notation. An arrow indicates proper inclusion, a bidirectional arrow indicates equality. It is still open whether OGDFex $_{a} \subseteq$ NUShBc, for $a \geq 3$.

tures is more restrictive than disallowing returning to correct conjectures in the TxtEx and in the TxtFex models, while the two restrictions are incomparable in the TxtBc case. On the other hand, disallowing inverted U's, disallowing returning to wrong overgeneralizing conjectures, and disallowing returning to overinclusive conjectures are equivalent to disallowing returning to correct conjectures for TxtEx. For TxtFex-identification, instead, disallowing returning to overgeneralizing conjectures is less restrictive than, equivalently, disallowing returning to correct or overinclusive conjectures, and disallowing inverted U's.

Also, while, for the second level, $\mathbf{T x t F e x}$, of the vacillatory hierarchy the necessity of returning to correct conjectures is avoidable by allowing infinitely many correct conjectures in the limit, the necessity of returning to wrong 
conjectures is not avoidable in this way: there are $\mathbf{T x t F e x}_{2}$-learnable classes that cannot be TxtBc-learned by any WrD- learner. This and the above observations may again suggest that freedom of returning to wrong abandoned conjectures is even more central, for full learning power, than freedom of returning to correct conjectures.

The above results are illustrated in Figure 1, where an arrow indicates proper inclusion, a double arrow indicates equality and the absence of (transitive chains of) arrows indicates incomparability, except that it is open whether, for $a \geq 3$, OGDFex $_{a} \subseteq$ NUShBc.

The following three questions are open:

(a) ConsWrDBc = WrDBc?

(b) ConsDecBc $=$ DecBc?

(c) For $a \geq 3$ or $a=*$, is OGDFex $_{a} \subseteq$ NUShBc?

Also, the question of how many non U-shaped features can be simultaneously satisfied has not been investigated in full detail, except for the case of coupling consistency with various non U-shapedness requirements.

\section{Acknowledgements}

We would like to thank Rolf Wiehagen for useful discussions. The referees of COLT 2005 and Information and Computation provided several helpful and thorough comments. A preliminary version of this paper appeared as [10].

\section{References}

[1] D. Angluin. Inductive inference of formal languages from positive data. Information and Control, 45:117-135, 1980.

[2] G. Baliga, J. Case, W. Merkle, and F. Stephan. Unlearning helps. In Ugo Montanari, José D. P. Rolim and Emo Welzl, editors, Automata, Languages and Programming, 27th International Colloquium, volume 1853 of Lecture Notes in Computer Science, pages 844-855. Springer-Verlag, 2000.

[3] G. Baliga, J. Case, W. Merkle, F. Stephan, and R. Wiehagen. When unlearning helps. Manuscript, 2005. Preliminary version of the paper appeared as [2].

[4] J. Bārzdiņš. Inductive inference of automata, functions and programs. In Int. Math. Congress, Vancouver, pages 771-776, 1974.

[5] L. Blum and M. Blum. Toward a mathematical theory of inductive inference. Information and Control, 28:125-155, 1975.

[6] M. Blum. A machine-independent theory of the complexity of recursive functions. Journal of the ACM, 14:322-336, 1967. 
[7] M. Bowerman. Starting to talk worse: Clues to language acquisition from children's late speech errors. In S. Strauss and R. Stavy, editors, U-Shaped Behavioral Growth. Developmental Psychology Series. Academic Press, New York, 1982.

[8] S. Carey. Face perception: Anomalies of development. In S. Strauss and R. Stavy, editors, U-Shaped Behavioral Growth, Developmental Psychology Series. Academic Press, New York, 1982.

[9] L. Carlucci, J. Case, S. Jain, and F. Stephan. U-shaped learning may be necessary. In S. Jain, H. U. Simon and E. Tomita, editors, Algorithmic Learning Theory, 16th International Conference (ALT' 05), volume 3734 of Lecture Notes in Artificial Intelligence, pages 241-255. Springer Verlag, 2005. (Longer version is available as Technical Report TRA11/04, School of Computing, National University of Singapore, Nov 2004).

[10] L. Carlucci, S. Jain, E. Kinber, and F. Stephan. Variations on U-shaped learning. In, P. Auer and R. Meir, editors, Proceedings, 18th Annual Conference on Learning Theory (COLT' 05), volume 3559 of Lecture Notes in Artificial Intelligence, pages 382-397. Springer Verlag, 2005.

[11] J. Case. The power of vacillation in language learning. SIAM Journal on Computing, 28(6):1941-1969, 1999.

[12] J. Case and C. Lynes. Machine inductive inference and language identification. In M. Nielsen and E. M. Schmidt, editors, Proceedings of the 9th International Colloquium on Automata, Languages and Programming, volume 140 of Lecture Notes in Computer Science, pages 107-115. Springer-Verlag, 1982.

[13] J. Case and C. Smith. Comparison of identification criteria for machine inductive inference. Theoretical Computer Science, 25:193-220, 1983.

[14] C. H. Cashon and L. B. Cohen. Beyond U-shaped development in infants' processing of faces: An information-processing account. Journal of Cognition and Development, 5(1):59-80, 2004.

[15] C. H. Cashon and L. B. Cohen. The construction, deconstruction and reconstruction of infant face perception. In A. Slater and O. Pascalis, editors, The development of face processing in infancy and early childhood, pages 55-58. NOVA Science Publishers, New York, 2003.

[16] M. Fulk. Prudence and other conditions on formal language learning. Information and Computation, 85:1-11, 1990.

[17] M. Fulk, S. Jain, and D. Osherson. Open problems in systems that learn. Journal of Computer and System Sciences, 49(3):589-604, 1994.

[18] E. M. Gold. Language identification in the limit. Information and Control, 10:447-474, 1967.

[19] J. Hopcroft and J. Ullman. Introduction to Automata Theory, Languages and Computation. Addison-Wesley, 1979. 
[20] K. P. Jantke and H.-R. Beick. Combining postulates of naturalness in inductive inference. Journal of Information Processing and Cybernetics (EIK), 17:465484, 1981.

[21] S. Kurtz and J. Royer. Prudence in language learning. In D. Haussler and L. Pitt, editors, Proceedings of the Workshop on Computational Learning Theory, pages 143-156. Morgan Kaufmann, 1988.

[22] S. Lange and R. Wiehagen. Polynomial time inference of arbitrary pattern languages. New Generation Computing, 8:361-370, 1991.

[23] M. Machtey and P. Young. An Introduction to the General Theory of Algorithms. North Holland, New York, 1978.

[24] G. Marcus, S. Pinker, M. Ullman, M. Hollander, T. Rosen, and F. Xu. Overregularization in Language Acquisition. Monographs of the Society for Research in Child Development, vol. 57, no. 4. University of Chicago Press, 1992. Includes commentary by Harold Clahsen.

[25] D. Osherson, M. Stob, and S. Weinstein. Systems that Learn: An Introduction to Learning Theory for Cognitive and Computer Scientists. MIT Press, 1986.

[26] K. Plunkett and V. Marchman. U-shaped learning and frequency effects in a multi-layered perceptron: implications for child language acquisition. Cognition, 38(1):43-102, 1991.

[27] K. Popper. The Logic of Scientific Discovery Harper Torch Books, New York, 1968.

[28] H. Rogers. Theory of Recursive Functions and Effective Computability. McGraw-Hill, 1967. Reprinted by MIT Press in 1987.

[29] S. Strauss and R. Stavy. U-Shaped Behavioral Growth. Developmental Psychology Series. Academic Press, New York, 1982.

[30] S. Strauss, R. Stavy, and N. Orpaz. The child's development of the concept of temperature. Manuscript, Tel-Aviv University, 1977.

[31] N. A. Taatgen and J. R. Anderson. Why do children learn to say broke? a model of learning the past tense without feedback. Cognition, 86(2):123-155, 2002 .

[32] R. Wiehagen and W. Liepe. Charakteristische Eigenschaften von erkennbaren Klassen rekursiver Funktionen. Journal of Information Processing and Cybernetics (EIK), 12:421-438, 1976. 